\title{
Simulation for Continuous-Time Markov Chains
}

\author{
Christel Baier $^{a}$, Joost-Pieter Katoen $^{b, \star}$, \\ Holger Hermanns $^{b}$ And Boudewijn Haverkort ${ }^{c}$ \\ ${ }^{a}$ Institut für Informatik I, University of Bonn \\ Römerstraße 164, D-53117 Bonn, Germany \\ ${ }^{c}$ Faculty of Computer Science, University of Twente \\ P.O. Box 217, 7500 AE Enschede, The Netherlands \\ ${ }^{b}$ Dept. of Computer Science, RWTH Aachen \\ Ahornstraße 55, D-52056 Aachen, Germany
}

\begin{abstract}
This paper presents a simulation preorder for continuoustime Markov chains (CTMCs). The simulation preorder is a conservative extension of a weak variant of probabilistic simulation on fully probabilistic systems, i.e., discrete-time Markov chains. The main result of the paper is that the simulation preorder preserves safety and liveness properties expressed in continuous stochastic logic (CSL), a stochastic branching-time temporal logic interpreted over CTMCs.
\end{abstract}

\section{Introduction}

To compare the stepwise behaviour of states in transition systems, simulation $(\sqsubseteq)$ and bisimulation relations $(\sim)$ have been widely considered [29,23]. Bisimulation relations are equivalences such that two bisimilar states exhibit identical stepwise behaviour. On the contrary, simulation relations are preorders on the state space such that if $s \sqsubseteq s^{\prime}$ ( " $s$ ' simulates $s$ ") state $s^{\prime}$ can mimic all stepwise behaviour of $s$; the converse, i.e., $s^{\prime} \sqsubseteq s$ is not guaranteed, so state $s^{\prime}$ may perform steps that cannot be matched by $s$. Thus, if $s \sqsubseteq s^{\prime}$ then every successor of $s$ has a corresponding, i.e., related successor of $s^{\prime}$, but the reverse does not necessarily hold. Simulation can be lifted to entire transition systems by comparing (according to ) their initial states. Simulation relations are often used for verification purposes to show that one system correctly implements another, more abstract system. One of the interesting aspects of simulation relations is that they allow a verification by "local" reasoning.

In the setting of model checking, (bi)simulation relations can be used to combat the well-known state-space explosion problem [14]. Here, bisimulation relations possess the so-called strong preservation property, whereas simulation possesses weak preservation. Strong preservation means: if $s \sim s^{\prime}$, then for all formulas $\Phi$ it follows $s \models \Phi$ iff $s^{\prime} \models \Phi$. This property holds, for instance, for CTL (and CTL*) and strong bisimulation [11]. The use of simulation relies on

\footnotetext{
* Contact author. Tel.: +31-53-4895675, fax: +31-53-4893247, e-mail: katoen@cs. utwente.nl.
} 
the preservation of certain classes of formulas, not of all formulas (such as for $\sim)$. For instance, if $s \sqsubseteq s^{\prime}$ then for all safety formulas $\Phi$ it follows that $s^{\prime} \models \Phi$ implies $s \models \Phi .^{1}$ Note that the converse, $s^{\prime} \not \models \Phi$, can not be used to deduce that $\Phi$ does not hold in the simulated state $s$; hence, the name weak preservation. As simulation equivalence - defined as mutual simulation of states - is coarser than bisimulation equivalence it yields a "better abstraction", i.e., a smaller quotient. Simulation relations are the basis for abstraction techniques where the rough idea is to replace the large system to be verified by a small abstract model and to model check the abstract system.

This paper studies a simulation preorder for continuous-time Markov chains (CTMCs) [27,34] and investigates the preservation of properties expressed in continuous stochastic logic (CSL) [3,6]. CTMCs are an important class of stochastic processes that are widely used in practice to determine system performance and dependability characteristics. CSL is a continuous probabilistic variant of CTL and includes means to express both transient and steady-state performance measures. For instance, it allows one to stipulate that the probability of reaching a certain set of goal-states within a specified real-valued time bound, provided that all paths to these states obey certain properties, is at least/at most some probability value. Model-checking algorithms for CSL have been presented in [6, $8]$, and prototypical software implementations are available: one based on sparse matrices [21] and a symbolic one based on multi-terminal BDDs [26]. Baier et al. [8] prove that lumping equivalence [12] - a continuous time variant of probabilistic bisimulation - preserves CSL; Desharnais and Panangaden [17] have recently shown the converse, namely that the equivalence induced by CSL implies lumping equivalence.

This paper proposes a novel simulation preorder $\left(\Xi_{m}\right)$ for CTMCs. This notion extends probabilistic simulation $\left(\Xi_{p}\right)$ on discrete-time Markov chains (DTMCs), as originally defined by Jonsson and Larsen [24]. The main result of the paper is that $\sqsubseteq_{m}$ weakly preserves CSL safety and liveness properties. This means that for $s \sqsubseteq_{m} s^{\prime}$ we have that $s^{\prime} \models \Phi_{\text {safe }}$ implies $s \models \Phi_{\text {safe }}$ for CSL safetyformula $\Phi_{\text {safe }}$, and that $s \models \Phi_{\text {live }}$ implies $s^{\prime} \models \Phi_{\text {live }}$ for CSL liveness-formula $\Phi_{\text {live }}$. As a consequence, the validity of safety formulas and the refutation of liveness formulas carries over from the abstract state $s^{\prime}$ (wrt. $\sqsubseteq_{m}$ ) to the concrete state $s$. This result can be used to verify CSL-formulas for a CTMC by verifying the same formulas on a smaller or simpler CTMC which is an abstraction of it.

Organisation of the paper. Section 2 introduces CTMCs, presents the simulation preorder for CTMCs and some of its elementary properties. Section 3 recalls CSL and introduces its safe and live fragments. Section 4 discusses weak preservation of CSL-formulas. Section 5 defines simulation equivalence and compares this to other equivalence notions. Section 6 discusses related work. Section 7 concludes the paper. Proofs of the main results are provided in the appendix.

\footnotetext{
${ }^{1}$ Safety formulas are here to be understood as arbitrary formulas in $\forall \mathrm{CTL}^{*}$, the restriction of $\mathrm{CTL}^{*}$ to universal path-quantifiers [13].
} 


\section{Simulation for CTMCs}

Fully probabilistic systems. Let $A P$ be a fixed, finite set of atomic propositions. A (labelled) fully probabilistic system (FPS) $\mathcal{D}$ is a tuple $(S, \mathbf{P}, L)$ where $S$ is a countable set of states, $\mathbf{P}: S \times S \rightarrow[0,1]$ is a probability matrix satisfying $\sum_{s^{\prime} \in S} \mathbf{P}\left(s, s^{\prime}\right) \in[0,1]$ for all $s \in S$, and $L: S \rightarrow 2^{A P}$ is a labelling function which assigns to each state $s \in S$ the set $L(s)$ of atomic propositions that are valid in $s$. If $\sum_{s^{\prime} \in S} \mathbf{P}\left(s, s^{\prime}\right)=1$ for all $s \in S$, then $\mathbf{P}(s, \cdot)$ (and $\mathcal{D}$ ) is called stochastic, otherwise it is called sub-stochastic. A (labelled) DTMC is an FPS with $\sum_{s^{\prime} \in S} \mathbf{P}\left(s, s^{\prime}\right) \in\{0,1\}$ for all $s \in S$.

Continuous-time Markov chains. A (labelled) CTMC $\mathcal{M}$ is a tuple $(S, \mathbf{R}, L)$ where $S$ and $L$ are as before, and $\mathbf{R}: S \times S \rightarrow \mathbb{R}_{\geqslant 0}$ is the rate matrix. (We adopt the same conventions as in [8,6], i.e., we do allow self-loops.) The exit rate $E(s)=\sum_{s^{\prime} \in S} \mathbf{R}\left(s, s^{\prime}\right)$ denotes that the probability of taking a transition from $s$ within $t$ time units equals $1-e^{-E(s) \cdot t}$. If $\mathbf{R}\left(s, s^{\prime}\right)>0$ for more than one state $s^{\prime}$, a race between the outgoing transitions from $s$ exists. That is, the probability $\mathbf{P}\left(s, s^{\prime}\right)$ of moving from $s$ to $s^{\prime}$ in a single step equals the probability that the delay of going from $s$ to $s^{\prime}$ "finishes before" the delays of any other outgoing transition from $s$; i.e., $\mathbf{P}\left(s, s^{\prime}\right)=\mathbf{R}\left(s, s^{\prime}\right) / E(s)$ if $E(s)>0$ and 0 otherwise.

Definition 1. For $C T M C \mathcal{M}=(S, \mathbf{R}, L)$, the embedded discrete-time Markov chain is given by $\operatorname{emb}(\mathcal{M})=(S, \mathbf{P}, L)$, where $\mathbf{P}\left(s, s^{\prime}\right)=\mathbf{R}\left(s, s^{\prime}\right) / E(s)$ if $E(s)>$ 0 , and $\mathbf{P}(s, s)=1$ and $\mathbf{P}\left(s, s^{\prime}\right)=0$ for $s \neq s^{\prime}$ if $E(s)=0$.

Note that, by definition, the embedded DTMC emb $(\mathcal{M})$ of any $\operatorname{CTMC} \mathcal{M}$ is stochastic.

Definition 2. For $C T M C \mathcal{M}=(S, \mathbf{R}, L)$ the uniformised $C T M C$ is given by unif $(\mathcal{M})=(S, \overline{\mathbf{R}}, L)$ where $\overline{\mathbf{R}}\left(s, s^{\prime}\right)=\mathbf{R}\left(s, s^{\prime}\right)$ for $s \neq s^{\prime}$ and $\overline{\mathbf{R}}(s, s)=\mathbf{R}(s, s)+$ $E-E(s)$ where constant $E \geqslant \max _{s \in S} E(s)$.

$E$ is called the uniformisation rate of $\mathcal{M}$, and is determined by the state with the shortest mean residence time, since $E \geqslant \max _{s \in S} E(s)$. All rates of self-loops in the CTMC $\mathcal{M}$ are "normalised" with respect to $E$, and hence the mean residence time is uniformly set to $1 / E$ in $\operatorname{unif}(\mathcal{M})$. In the literature $[19,22]$, uniformisation is often defined by transforming $\mathrm{CTMC} \mathcal{M}$ into the DTMC emb(unif $(\mathcal{M}))$. For technical convenience, we here define uniformisation as a transformation from CTMCs to CTMCs basically by adding self-loops to slower states (as e.g. in [31]).

Simulation for fully probabilistic systems. For labelled transition systems, state $s^{\prime}$ simulates $s$ if for each successor $t$ of $s$ there is a successor $t^{\prime}$ of $s^{\prime}$ that simulates $t$. Simulation of two states is thus defined in terms of simulation of their successor states. In the probabilistic setting, the target of a transition is in fact a probability distribution, and thus, the simulation relation $\sqsubseteq$ needs to be lifted from states to distributions. This can be done using weight functions [24]. For countable set $X$, let $\operatorname{Dist}(X)$ denote the collection of all, possibly sub-stochastic, distributions on $X$. 
Definition 3. Let $\mu \in \operatorname{Dist}(X)$ and $\mu^{\prime} \in \operatorname{Dist}(Y)$ and $\sqsubseteq \subseteq X \times Y$. Then $\mu \preceq \mu^{\prime}$ iff there exists a weight function $\Delta: X \times Y \rightarrow[0,1]$ for $\sqsubseteq$ such that:

1. $\Delta(x, y)>0$ implies $x \sqsubseteq y$

2. $\mu(x)=K_{1} \cdot \sum_{y \in Y} \Delta(x, y)$ for any $x \in X$

3. $\mu^{\prime}(y)=K_{2} \cdot \sum_{x \in X} \Delta(x, y)$ for any $y \in Y$,

where $K_{1}=\sum_{x \in X} \mu(x)$ and $K_{2}=\sum_{y \in Y} \mu^{\prime}(y)$.

Intuitively, a weight function $\Delta$ shows how the probability $\mu(x)$ can be distributed among the related states $y$ such that $\mu^{\prime}(y)$ equals the total amount of probability it gets distributed by $\Delta$. (Note that $K_{1}=K_{2}=1$ for stochastic $\mu$ and $\mu^{\prime}$.) $\Delta$ is a probability distribution on $X \times Y$ such that the probability to select $(x, y)$ with $x \sqsubseteq y$ is one. In addition, the probability to select an element in $\sqsubseteq$ whose first component is $x$ equals $\mu(x)$, and the probability to select an element in $\sqsubseteq$ whose second component is $y$ equals $\mu^{\prime}(y)$.

Example 1. Let $X=\{s, t\}$ and $Y=\{u, v, w\}$ with $\mu(s)=\frac{2}{9}, \mu(t)=\frac{2}{3}$ and $\mu^{\prime}(u)=\frac{1}{3}, \mu^{\prime}(v)=\frac{4}{9}$ and $\mu^{\prime}(w)=\frac{1}{9} ; K_{1}=K_{2}=\frac{8}{9}$. Note that $\mu$ and $\mu^{\prime}$ are both sub-stochastic. Let $\sqsubseteq=(X \times Y) \backslash\{(s, w)\}$. We have $\mu \preceq \mu^{\prime}$, as weight function $\Delta$ defined by $\Delta(s, u)=\Delta(s, v)=\Delta(t, w)=\frac{1}{8}, \Delta(t, v)=\frac{3}{8}$ and $\Delta(t, u)=\frac{1}{4}$ satisfies the constraints of Def. 3.

For fully probabilistic systems we consider a slight variant of probabilistic simulation by Jonsson and Larsen [24]:

Definition 4. For FPS $(S, \mathbf{P}, L)$, let $\sqsubseteq_{p}$ be the coarsest binary relation on the state space $S$ such that for all $s_{1} \sqsubseteq_{p} s_{2}$ :

1. $L\left(s_{1}\right)=L\left(s_{2}\right)$ and

2. $\mathbf{P}\left(s_{1}, \cdot\right) \preceq \mathbf{P}\left(s_{2}, \cdot\right)$.

Relation $\sqsubseteq_{p}$ is symmetric if the transition probabilities are stochastic [24]. In this case, the simulation preorder agrees with probabilistic bisimulation [28]. Thus, for instance, $\sqsubseteq_{p}$ and probabilistic bisimulation $\sim_{p}$ coincide for an embedded DTMC of a CTMC.

Simulation for CTMCs. For CTMCs we modify $\sqsubseteq_{p}$ such that timing aspects are incorporated. Intuitively, we intend a simulation preorder to ensure that $s_{2}$ simulates $s_{1}$ iff (i) $s_{2}$ is "faster than" $s_{1}$ and (ii) the time-abstract behaviour of $s_{2}$ simulates that of $s_{1}$. An obvious attempt in this direction would be to refine Def. 4 by demanding that in addition to $\mathbf{P}\left(s_{1}, \cdot\right) \preceq \mathbf{P}\left(s_{2}, \cdot\right)$, we have $E\left(s_{1}\right) \leqslant E\left(s_{2}\right)$, i.e., $s_{2}$ should be on average at least as fast as $s_{1}$. However, such an approach turns out not to be very useful, as this would coincide with lumping equivalence [12] for uniformised CTMCs. Therefore, we present a more involved definition, which - in return - also enables a more radical state-space aggregation, since it incorporates a notion of stuttering $[11,36]$. 
Definition 5. Let $\mathcal{M}=(S, \mathbf{R}, L)$ be a CTMC. Relation $\sqsubseteq \subseteq S \times S$ is a simulation iff for all states $s_{1}, s_{2} \in S$ with $s_{1} \sqsubseteq s_{2}$ we have that $L\left(s_{1}\right)=L\left(s_{2}\right)$ and there exist functions $\Delta: S \times S \rightarrow[0,1], \delta_{i}: S \rightarrow[0,1]$ and sets $U_{i}, V_{i} \subseteq S$ (for $i=1,2)$ with:

$$
\begin{aligned}
& U_{i}=\left\{u_{i} \in S \mid \mathbf{R}\left(s_{i}, u_{i}\right)>0 \wedge \delta_{i}\left(u_{i}\right)>0\right\} \text { and } \\
& V_{i}=\left\{v_{i} \in S \mid \mathbf{R}\left(s_{i}, v_{i}\right)>0 \wedge \delta_{i}\left(v_{i}\right)<1\right\}
\end{aligned}
$$

such that:

1. $v_{1} \sqsubseteq s_{2}$ for any $v_{1} \in V_{1}$ and $s_{1} \sqsubseteq v_{2}$ for any $v_{2} \in V_{2}$,

2. $\Delta\left(u_{1}, u_{2}\right)>0$ implies $u_{1} \in U_{1}, u_{2} \in U_{2}$ and $u_{1} \sqsubseteq u_{2}$,

3. $K_{1} \cdot \sum_{u_{2} \in U_{2}} \Delta\left(w, u_{2}\right)=\delta_{1}(w) \cdot \mathbf{P}\left(s_{1}, w\right)$ and

$K_{2} \cdot \sum_{u_{1} \in U_{1}} \Delta\left(u_{1}, w\right)=\delta_{2}(w) \cdot \mathbf{P}\left(s_{2}, w\right)$, for all $w \in S$, and

4. $K_{1} \cdot E\left(s_{1}\right) \leqslant K_{2} \cdot E\left(s_{2}\right)$

where $K_{i}=\sum_{u_{i} \in U_{i}} \delta_{i}\left(u_{i}\right) \cdot \mathbf{P}\left(s_{i}, u_{i}\right)$ for $i=1,2$.

Definition 6. The simulation relation $\sqsubseteq_{m}$ is defined by: $s_{1} \sqsubseteq_{m} s_{2}$ iff there exists a simulation $\sqsubseteq$ such that $s_{1} \sqsubseteq s_{2}$.

The successor states of $s_{i}$ are grouped into the subsets $U_{i}$ and $V_{i}$. Although we do not require that $U_{i}$ and $V_{i}$ are disjoint, to understand the definition first consider $U_{i} \cap V_{i}=\varnothing$. (The fact that we allow a non-empty intersection has technical reasons that will be explained later). $K_{i}$ denotes the total probability to move from $s_{i}$ within one transition to a state in $U_{i}$. Vice versa, with probability $1-K_{i}$, in state $s_{i}$ a transition to some state in $V_{i}$ is made (cf. Fig. 1). The first

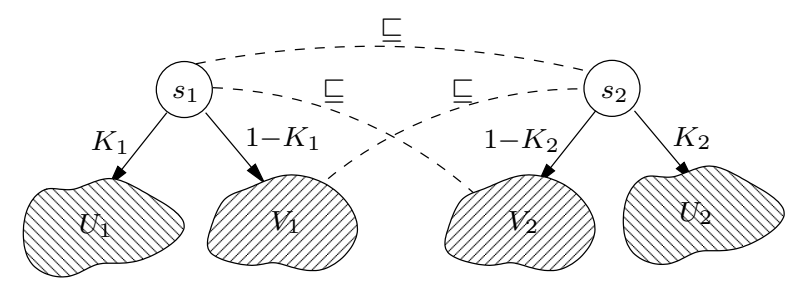

Fig. 1. Simulation scenario

condition states that the grouping of successor states into $V_{i}$ and $U_{i}$ is such that any state in $V_{2}$ simulates $s_{1}$ and that $s_{2}$ simulates any state in $V_{1}$. The second and third condition require the existence of a weight function $\Delta$ that relates the conditional probabilities to move from $s_{1}$ to a $U_{1}$-state and the conditional probabilities for $s_{2}$ to move to a $U_{2}$-state. Thus, $\Delta$ is a weight function for the probability distributions $\delta_{i}(\cdot) \cdot \mathbf{P}\left(s_{i}, \cdot\right) / K_{i}$. Finally, the fourth condition states 
that $s_{2}$ is "faster than" $s_{1}$ in the sense that the total rate to move from $s_{2}$ to a $U_{2}$-state is at least the total rate to move from $s_{1}$ to a $U_{1}$-state.

Intuitively, we interpret the moves from $s_{i}$ to a $V_{i}$-state as silent transitions (i.e., a $\tau$-transition for action-labeled transition systems). The first condition in Def. 5 guarantees that any such transition is a "stutter" step. Vice versa, the transitions from $s_{i}$ to a $U_{i}$-state are considered as observable moves.

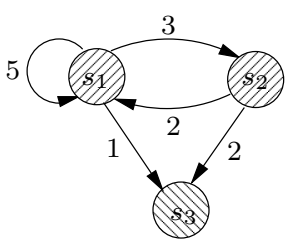

(1)

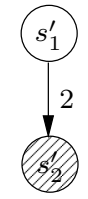

(2)

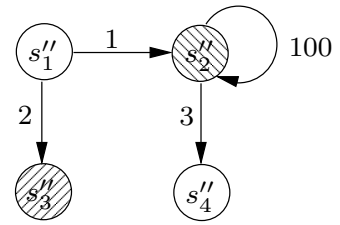

(3)

Fig. 2. Some examples of simulation refinement: $s_{1} \sqsubseteq_{m} s_{1}^{\prime}$ and $s_{1}^{\prime} \sqsubseteq_{m} s_{1}^{\prime \prime}$

Example 2. Consider the three CTMCs depicted in Fig. 2 where states $s_{1}, s_{2}, s_{1}^{\prime}$, $s_{1}^{\prime \prime}$ and $s_{2}^{\prime \prime}$ are labelled with proposition $a$, and the other states by $b$. We have $s_{1} \sqsubseteq_{m} s_{1}^{\prime}$, since there exists a relation $\sqsubseteq=\left\{\left(s_{1}, s_{1}^{\prime}\right),\left(s_{3}, s_{2}^{\prime}\right),\left(s_{2}^{\prime}, s_{3}\right),\left(s_{2}, s_{1}^{\prime}\right)\right\}$ with $U_{1}=\left\{s_{3}\right\}, V_{1}=\left\{s_{1}, s_{2}\right\}, K_{1}=\frac{1}{9}, \delta_{1}\left(s_{3}\right)=1$ and 0 otherwise, $U_{2}=\left\{s_{2}^{\prime}\right\}$, $V_{2}=\varnothing, \delta_{2}\left(s_{2}^{\prime}\right)=1$ and 0 otherwise, $K_{2}=1$, and $\Delta\left(s_{3}, s_{2}^{\prime}\right)=\Delta\left(s_{2}^{\prime}, s_{3}\right)=1$ and 0 otherwise. (In the pictorial representation, the elements of $U_{i}$ and $V_{i}$ are indicated by the same patterns used in Fig. 1 for $U_{i}$ and $V_{i}$ ). It is not difficult to check that indeed all constraints of Def. 5 are fulfilled, e.g., for the fourth constraint we obtain $\frac{1}{9} \cdot 9 \leqslant 1 \cdot 2$. Note that $s_{1} \unrhd_{m} s_{2}$ if $\mathbf{R}\left(s_{2}, s_{3}\right)$ would exceed 2 (rather than being equal to 2), since then $s_{2} \sqsubseteq s_{1}^{\prime}$ can no longer be established.

We further have $s_{1}^{\prime} \sqsubseteq_{m} s_{1}^{\prime \prime}$ since there exists a relation $\sqsubseteq=\left\{\left(s_{1}^{\prime}, s_{1}^{\prime \prime}\right),\left(s_{1}^{\prime}, s_{2}^{\prime \prime}\right)\right.$, $\left.\left(s_{2}^{\prime}, s_{3}^{\prime \prime}\right),\left(s_{3}^{\prime \prime}, s_{2}^{\prime}\right),\left(s_{2}^{\prime}, s_{4}^{\prime \prime}\right),\left(s_{4}^{\prime \prime}, s_{2}^{\prime}\right)\right\}$ with $U_{1}=\left\{s_{2}^{\prime}\right\}, V_{1}=\varnothing, K_{1}=1$, and $\delta_{1}\left(s_{2}^{\prime}\right)=$ 1 and 0 otherwise, $U_{2}=\left\{s_{3}^{\prime \prime}\right\}, V_{2}=\left\{s_{2}^{\prime \prime}\right\}, \delta_{2}\left(s_{3}^{\prime \prime}\right)=1$ and 0 otherwise, $K_{2}=\frac{2}{3}$ and $\Delta\left(s_{3}^{\prime \prime}, s_{2}^{\prime}\right)=\Delta\left(s_{2}^{\prime}, s_{3}^{\prime \prime}\right)=1$. It is straightforward to check that indeed all constraints of Def. 5 are fulfilled.

In the examples so far, we have used the special case where $\delta_{i}(s) \in\{0,1\}$ for any state $s$. In this case, $\delta_{i}$ is the characteristic function of $U_{i}$, and the sets $U_{i}$ and $V_{i}$ are disjoint. In general, though, things are more complicated and we need to construct $U_{i}$ and $V_{i}$ using fragments of states. That is, we deal with functions $\delta_{i}$ where $0 \leqslant \delta_{i}(s) \leqslant 1$. Intuitively, the $\delta_{i}(s)$-fragment of state $s$ belongs to $U_{i}$, while the remaining part (the $\left(1-\delta_{i}(s)\right)$-part) of $s$ belongs to $V_{i}$. The use of fragments of states is exemplified in the following example.

Example 3. Consider the two CTMCs depicted in Fig. 3. where $L\left(s_{1}\right)=L\left(s_{3}\right)=$ $L\left(s_{1}^{\prime}\right)=L\left(s_{3}^{\prime}\right)=\{a\}$; the other states are labelled by $b$. Intuitively, $s_{1}$ is "slower than" $s_{1}^{\prime}$. However, when we require the sets $U_{i}, V_{i}$ in Def. 5 to be disjoint, then $s_{1} \nsubseteq_{m} s_{1}^{\prime}$. This can be seen as follows. We have $s_{1} \nsubseteq_{m} s_{3}^{\prime}$ (and hence, $V_{2}=\varnothing$ ) 


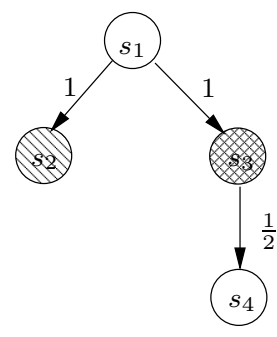

(1)

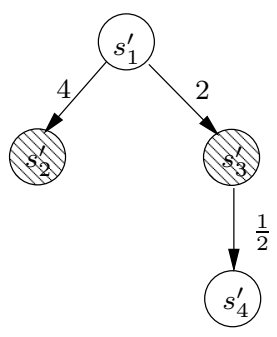

(2)

Fig. 3. An example of simulation using fragments of states

as $s_{1}$ moves with rate 1 to a $b$-state while the total rate for $s_{3}^{\prime}$ to move to a $b$-state is smaller (i.e., $\frac{1}{2}$ ). Hence, the only chance to define the components in Def. 5 is $V_{2}=\varnothing$ and $U_{2}=\left\{s_{2}^{\prime}, s_{3}^{\prime}\right\}$. Because $s_{3}^{\prime}$ and $s_{2}$ are not comparable with the simulation order (as they have different labels), we would have to define $U_{1}=\left\{s_{2}, s_{3}\right\}$ and $V_{1}=\varnothing$. But then, the weight-function condition is violated because $s_{1}$ moves with probability $\frac{1}{2}$ to a $b$-state while the probability for $s_{1}^{\prime}$ to reach a $b$-state within one step is $\frac{2}{3}$.

On the other hand, when we allow $s_{3}$ to be splitted: one half of $s_{3}$ belongs to $U_{1}$, one half to $V_{1}$, i.e., $\delta_{1}\left(s_{3}\right)=\frac{1}{2}$ and $U_{1}=\left\{s_{2}, s_{3}\right\}, V_{1}=\left\{s_{3}\right\}$ then we get that with $U_{2}=\left\{s_{2}^{\prime}, s_{3}^{\prime}\right\}, V_{2}=\varnothing$ and $\Xi_{m}=\left\{\left(s_{1}, s_{1}^{\prime}\right),\left(s_{2}, s_{2}^{\prime}\right),\left(s_{3}, s_{1}^{\prime}\right),\left(s_{4}, s_{4}^{\prime}\right),\left(s_{2}, s_{4}^{\prime}\right)\right\}$ the conditional probabilities for the $U_{i}$-states are related via $\preceq$. Note that $K_{1}=$ $\frac{1}{2}+\frac{1}{4}=\frac{3}{4}, K_{2}=1$ and $\Delta\left(s_{2}, s_{2}^{\prime}\right)=\frac{2}{3}, \Delta\left(s_{3}, s_{3}^{\prime}\right)=\frac{1}{3}$.

Remark 1. It is interesting to observe what happens if $s_{1} \sqsubseteq m s_{2}$ and one of the states is absorbing. If $s_{2}$ is absorbing (i.e., $E\left(s_{2}\right)=0$ ) then $K_{1} \cdot E\left(s_{1}\right)=0$. Hence, either $s_{1}$ has to be absorbing or $K_{1}=0$. In the latter case, we have $\delta_{1}\left(u_{1}\right)=0$ for all $u_{1} \in U_{1}$ (by condition 3. in Def. 5), i.e., all successor states of $s_{1}$ belong to $V_{1}$ and are simulated by $s_{2}$ (by condition 2 . in Def. 5 ). Vice versa, for any state $u_{2} \in U_{2}$ :

$$
0<\delta_{2}\left(u_{2}\right) \cdot \mathbf{P}\left(s_{2}, u_{2}\right)=\sum_{u_{1} \in U_{1}} \Delta\left(u_{1}, u_{2}\right)
$$

Thus, $\Delta\left(u_{1}, u_{2}\right)>0$ for some $u_{1} \in U_{1}$. In particular, if $U_{2} \neq \varnothing$ then $U_{1} \neq \varnothing$, which implies that $s_{1}$ is non-absorbing. This shows that, if $s_{1}$ is absorbing then all successor states of $s_{2}$ belong to $V_{2}$ and simulate $s_{1}$ (by condition 2. of Def. 5 ).

The observation that an absorbing state $s_{1}$ is simulated by any state $s_{2}$ with the same labeling is natural for any type of simulation that abstracts from silent moves. The observation that any state $s_{2}$ which simulates an absorbing state $s_{1}$ can only perform stutter steps (non-observable transitions) can be viewed as the probabilistic counterpart to divergence for non-probabilistic systems. Note that in absorbing states of a CTMC just time advances.

Lemma 1. $\sqsubseteq_{m}$ is a preorder. 
Lemma 2. For $C T M C \mathcal{M}=(S, \mathbf{R}, L)$ and $s_{1}, s_{2} \in S$ we have:

$$
s_{1} \sqsubseteq_{m}^{\mathcal{M}} s_{2} \text { if and only if } s_{1} \sqsubseteq_{m}^{\text {unif( }(\mathcal{M})} s_{2} .
$$

Here, the superscript of the simulation preorder indicates the CTMC on which it is considered. The proofs of these facts are in the appendix; we note here that the proof of Lemma 2 relies on the fact that sets $U_{i}$ and $V_{i}$ may overlap.

\section{Safe and Live CSL}

This section recapitulates the logic CSL and discusses two distinguished subsets of the logic that will in the sequel be shown to be weakly preserved by our simulation.

Paths in CTMCs. A path through a CTMC is an alternating sequence $\sigma=$ $s_{0} t_{0} s_{1} t_{1} s_{2} \ldots$ with $\mathbf{R}\left(s_{i}, s_{i+1}\right)>0$ and $t_{i} \in \mathbb{R}_{>0}$ for all $i .^{2}$ The time stamps $t_{i}$ denote the amount of time spent in state $s_{i}$. Let Path denote the set of paths through $\mathcal{M} . \sigma[i]$ denotes the $(i+1)$ th state of $\sigma$, i.e. $\sigma[i]=s_{i+1} . \sigma @ t$ denotes the state of $\sigma$ occupied at time $t$, i.e. $\sigma @ t=\sigma[i]$ with $i$ the smallest index such that $t \leqslant \sum_{j=0}^{i} t_{j}$. Let $\operatorname{Pr}_{s}$ denote the unique probability measure on sets of paths that start in $s$ (for a definition of the Borel space see [6]).

Continuous Stochastic Logic. CSL [6] is a branching-time temporal logic à la CTL where the state-formulas are interpreted over states of a CTMC and the path-formulas are interpreted over paths in a CTMC. CSL is a variant of the (equally named) logic by Aziz et al. [3] and incorporates (i) an operator to refer to the probability of the occurrence of particular paths, similar to PCTL [20], a (ii) real-time until-operator, like in TCTL [1], and (iii) a steady-state operator [6]. In this paper, we focus on a fragment of CSL (denoted $\mathrm{CSL}^{-}$), distinguished in that we do not consider the next step and steady-state operator. (For simplicity, we also only consider time-intervals of the form $[0, t]$.) The omission of these operators will be justified later on. Besides the usual strong until-operator we incorporate a weak until-operator that will be used in the classification of safety and liveness properties. These properties are subjects of the weak preservation results we aim to establish.

Recall that $A P$ is the set of atomic propositions. Let $a \in A P, p \in[0,1]$ and $\unlhd \in\{\leqslant, \geqslant\}$ and $t \in \mathbb{R}_{\geqslant 0}$ (or $\infty$ ). The syntax of $\mathrm{CSL}^{-}$is:

$$
\Phi::=a|\Phi \wedge \Phi| \neg \Phi\left|\mathcal{P}_{\unlhd p}\left(\Phi \mathcal{U}^{\leqslant t} \Phi\right)\right| \mathcal{P}_{\unlhd p}\left(\Phi \mathcal{W}^{\leqslant t} \Phi\right) .
$$

$\mathcal{P}_{\triangleleft p}(\varphi)$ asserts that the probability measure of the paths satisfying $\varphi$ meets the bound given by $\unlhd p$. The operator $\mathcal{P}_{\unlhd p}($.$) replaces the usual (fair) CTL path$ quantifiers $\exists$ and $\forall$. The path-formula $\Phi \mathcal{U} \leqslant t ~ \Psi$ asserts that $\Psi$ is satisfied at some

\footnotetext{
${ }^{2}$ For paths that end in an absorbing state $s_{k}$ we assume a path to be represented as an infinite sequence $s_{0} t_{0} s_{1} \ldots t_{k-1} s_{k} 1 s_{k} 1 s_{k} 1 \ldots$
} 
time instant before $t$ and that at all preceding time instants $\Phi$ holds (strong until). The weak until-operator $\mathcal{W}$ differs in that we do not require that $\Psi$ eventually becomes true, i.e., $\Phi \mathcal{W}^{\leqslant t} \Psi$ means $\Phi \mathcal{U}^{\leqslant t} \Psi$ unless always $\Phi$ in the time-interval $[0, t]$ holds.

Semantics. The semantics of CSL for the boolean operators is identical to that for CTL and is omitted here. For the remaining state-formulas [6]:

$$
s \models \mathcal{P}_{\unlhd p}(\varphi) \text { iff } \operatorname{Prob}(s, \varphi) \unlhd p
$$

for path-formula $\varphi$. Here, $\operatorname{Prob}(s, \varphi)=\operatorname{Pr}_{s}\{\sigma \in \operatorname{Path} \mid \sigma \models \varphi\}$. The semantics of $\mathcal{U}^{\leqslant t}$ is defined by:

$$
\sigma \models \Phi \mathcal{U}^{\leqslant t} \Psi \text { iff } \exists x \leqslant t .(\sigma @ x \models \Psi \wedge \forall y<x . \sigma @ y \models \Phi) .
$$

Note that the standard (i.e., untimed) until operator is obtained by taking $t$ equal to $\infty$. The semantics of the weak until operator is defined by:

$$
\sigma \models \Phi \mathcal{W}^{\leqslant t} \Psi \text { iff }(\forall x \leqslant t . \sigma @ x \models \Phi) \vee \sigma \models \Phi \mathcal{U}^{\leqslant t} \Psi .
$$

The other boolean connectives are derived in the usual way, i.e., tt $=a \vee \neg a$, ff $=$ $\neg \mathrm{tt}, \Phi_{1} \vee \Phi_{2}=\neg\left(\neg \Phi_{1} \wedge \neg \Phi_{2}\right)$, and $\Phi_{1} \rightarrow \Phi_{2}=\neg \Phi_{1} \vee \Phi_{2}$. Temporal operators like $\diamond, \square$ and their real-time variants $\diamond \leqslant t$ or $\square^{\leqslant t}$ can be derived, e.g.

$$
\mathcal{P}_{\unlhd p}\left(\diamond^{\leqslant t} \Phi\right)=\mathcal{P}_{\unlhd p}\left(\operatorname{tt} \mathcal{U}^{\leqslant t} \Phi\right) \text { and } \mathcal{P}_{\unlhd p}\left(\square^{\leqslant t} \Phi\right)=\mathcal{P}_{\unlhd p}\left(\Phi \mathcal{W}^{\leqslant t} \mathrm{ff}\right) .
$$

For instance, if error is an atomic proposition that characterizes all states where a system error has occurred then $\mathcal{P}_{<0.001}(\diamond \leqslant 4$ error $)$ asserts that the probability for a system error within 4 time units is smaller than 0.001 .

The until-operator and the weak until-operator are closely related. For any state $s$ and $\mathrm{CSL}^{-}$-formula $\Phi$ and $\Psi$ we have:

$$
\begin{gathered}
\operatorname{Prob}\left(s, \Phi \mathcal{U}^{\leqslant t} \Psi\right)=1-\operatorname{Prob}\left(s,(\neg \Psi) \mathcal{W}^{\leqslant t}(\neg \Phi \wedge \neg \Psi)\right) \\
\operatorname{Prob}\left(s, \Phi \mathcal{W}^{\leqslant t} \Psi\right)=1-\operatorname{Prob}\left(s,(\neg \Psi) \mathcal{U}^{\leqslant t}(\neg \Phi \wedge \neg \Psi)\right)
\end{gathered}
$$

Hence, the following two formulas are equivalent:

$$
\mathcal{P}_{\geqslant p}\left(\Phi \mathcal{W}^{\leqslant t} \Psi\right) \text { and } \mathcal{P}_{\leqslant 1-p}\left((\neg \Psi) \mathcal{U}^{\leqslant t}(\neg \Phi \wedge \neg \Psi)\right) .
$$

A similar equivalence holds when the weak until- and the until-operator are exchanged. Note that a path satisfies $\neg\left((\neg \Phi) \mathcal{U}^{\leqslant t}(\neg \Psi)\right)$ if $\Psi$ always holds, a requirement that is released as soon as $\Phi$ becomes valid.

CSL safety and liveness properties. For the weak preservation results we distinguish between safety ("something bad never happens") and liveness ("something good will eventually happen") properties. In order to do so, negations may only be attached to atomic propositions. The syntax of $\mathrm{CSL}_{\text {safe }}$, the set of safety formulas, is defined by:

$$
\Phi::=a|\neg a| \Phi \wedge \Phi|\Phi \vee \Phi| \mathcal{P}_{\geqslant p}\left(\Phi \mathcal{W}^{\leqslant t} \Phi\right) \quad \mid \mathcal{P}_{\leqslant p}\left(\neg \Phi \mathcal{U}^{\leqslant t} \neg \Phi\right) .
$$


An example CSL safety formula is $\mathcal{P}_{\geqslant 0.99}(\square \leqslant 100 \neg$ error $)$ expressing that with probability at least 0.99 no error will occur in the next hundred time units. The syntax of $\mathrm{CSL}_{\text {live }}$, the set of liveness formulas, is defined by:

$$
\Phi::=a|\neg a| \Phi \wedge \Phi|\Phi \vee \Phi| \mathcal{P}_{\geqslant p}\left(\Phi \mathcal{U}^{\leqslant t} \Phi\right) \mid \mathcal{P}_{\leqslant p}\left(\neg \Phi \mathcal{W}^{\leqslant t} \neg \Phi\right) .
$$

As a result of the aforementioned relationship between $\mathcal{U}$ and $\mathcal{W}$ (cf. equations (1) and (2)), there is a duality between safety and liveness properties for CSL, i.e., for any formula $\Phi_{\text {safe }}$ there is a liveness property equivalent to $\neg \Phi_{\text {safe }}$, and the same applies to liveness property $\Phi_{\text {live }}$.

Next and steady state. Neither the next operator $\mathcal{P}_{\unlhd p}(X \Phi)$, nor the steady-state operator $\mathcal{S}_{\unlhd p}(\Phi)$ of [6] can become part of a CSL fragment that enables a weak preservation result for $\sqsubseteq_{m}$. This is shown by the following example.

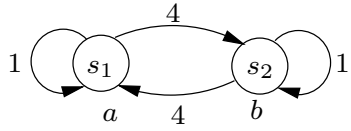

(1)

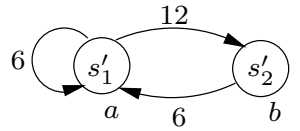

(2)

Fig. 4. Next and steady state behaviour is not preserved by $\sqsubseteq_{m}$.

Example 4. Consider the two CTMCs depicted in Fig. 4, where each state is decorated with the atomic propositions valid in the respective state. We have $s_{1} \sqsubseteq_{m} s_{1}^{\prime}$ and $s_{2} \sqsubseteq_{m} s_{2}^{\prime}$. The steady-state (or long-run) probability $\pi\left(s_{i}\right)$ of being in state $s_{i}$ is spread evenly among $s_{1}$ and $s_{2}$, whereas it is spread unevenly among $s_{1}^{\prime}$ and $s_{2}^{\prime} ; s_{1}^{\prime}$ is less likely than $s_{2}^{\prime}$. Concretely $\pi\left(s_{1}\right)=\pi\left(s_{2}\right)=\frac{1}{2}$ but $\pi\left(s_{1}^{\prime}\right)=\frac{1}{3}$ and $\pi\left(s_{2}^{\prime}\right)=\frac{2}{3}$. As a consequence, $s_{1} \models \mathcal{S}_{\geqslant 0.5}(a)$, but $s_{1}^{\prime} \not \models \mathcal{S}_{\geqslant 0.5}(a)$. On the other hand, $s_{2} \models \mathcal{S}_{\leqslant 0.5}(b)$, while $s_{2}^{\prime} \not \models \mathcal{S}_{\leqslant 0.5}(b)$. Furthermore, we have that $s_{1} \models \mathcal{P}_{\leqslant 0.2}(X a)$ and $s_{2} \models \mathcal{P}_{\geqslant 0.2}(X b)$, but $s_{1}^{\prime} \not \models \mathcal{P}_{\leqslant 0.2}(X a)$ and $s_{2}^{\prime} \not \mathcal{P}_{\geqslant 0.2}(X b)$.

The fact that the steady-state operator is not compatible with our simulation relation can be viewed as a specific instance of the well-known phenomenon that CTMCs cannot be ordered according to their steady-state performance $[33,10]$.

\section{Weak Preservation}

This section is devoted to the main result of the paper: weak preservation of the two CSL fragments $\mathrm{CSL}_{\text {safe }}$ and $\mathrm{CSL}_{\text {live }}$ with respect to $\sqsubseteq_{m}$. To arrive there, requires to establish some crucial observations.

For a given CTMC $\mathcal{M}$ we first remark that the probability measures on CTMC $\mathcal{M}$ agree with those on the uniformised CTMC unif $(\mathcal{M})$. For arbitrary CSL path-formula $\varphi$ we have: 
Lemma 3. $\operatorname{Pr}_{s}^{\mathcal{M}}\{\sigma \in \operatorname{Path} \mid \sigma \models \varphi\}=\operatorname{Pr}_{s}^{u n i f(\mathcal{M})}\{\sigma \in \operatorname{Path} \mid \sigma \models \varphi\}$.

The above lemma implies that CSL satisfaction on $\mathcal{M}$ agrees with CSL satisfaction on unif $(\mathcal{M})$. We thus may safely assume that the exit rate of each state equals $E$.

Theorem 1. For state $s_{1}, s_{2}$ :

1. for $C S L_{\text {safe }}$-formula $\Phi_{\text {safe }}: s_{1} \sqsubseteq_{m} s_{2} \Longrightarrow\left(s_{2} \models \Phi_{\text {safe }} \Longrightarrow s_{1} \models \Phi_{\text {safe }}\right)$.

2. for $C S L_{\text {live }}$-formula $\Phi_{\text {live }}: s_{1} \sqsubseteq_{m} s_{2} \Longrightarrow\left(s_{2} \not \models \Phi_{\text {live }} \Longrightarrow s_{1} \not \models \Phi_{\text {live }}\right)$.

Proof. It is first proven that sets $\operatorname{Sat}\left(\Phi_{\text {safe }}\right)$ are upward-closed, i.e., if $s_{1} \sqsubseteq_{m} s_{2}$ and $s_{1} \in \operatorname{Sat}\left(\Phi_{\text {safe }}\right)$ then $s_{2} \in \operatorname{Sat}\left(\Phi_{\text {safe }}\right)$. This is not involved and omitted here. The proof is then by induction on the formula, where the interesting cases $\left(\mathcal{U}^{\leqslant t}\right.$ and $\left.\mathcal{W}^{\leqslant t}\right)$ use Lemma 4 below. The statement for the $\mathrm{CSL}_{\text {live }}$-formulas follows then by duality of the weak until- and until-operator.

The proof of the above theorem requires to establish the following fact (Lemma 4):

$$
s_{1} \sqsubseteq_{m} s_{2} \text { implies } \operatorname{Prob}\left(s_{1}, \Phi_{1} \mathcal{U}^{\leqslant t} \Phi_{2}\right) \leqslant \operatorname{Prob}\left(s_{2}, \Phi_{1} \mathcal{U}^{\leqslant t} \Phi_{2}\right),
$$

where sets $\operatorname{Sat}\left(\Phi_{i}\right)=\left\{s \in S \mid s \models \Phi_{i}\right\}$ are upward-closed. The initial proof idea for this fact is to resort to the embedded uniformised CTMC of $\mathcal{M}$, using the result that:

$$
\operatorname{Prob}^{\mathcal{M}}\left(s_{1}, \Phi_{1} \mathcal{U}^{\leqslant t} \Phi_{2}\right)=e^{-E \cdot t} \cdot \sum_{k=0}^{\infty} \frac{(E \cdot t)^{k}}{k !} \cdot \operatorname{Prob}^{\mathcal{D}}\left(s_{1}, \Phi_{1} \mathcal{U}^{\leqslant k} \Phi_{2}\right),
$$

where $\mathcal{D}=\operatorname{emb}(\operatorname{unif}(\mathcal{M}))$ and $\Phi_{1} \mathcal{U} \leqslant k \Phi_{2}$ means that $\Phi_{2}$ can be reached within at most $k$ steps via a $\Phi_{1}$-path (for natural $k$ ) [20]. The advantage of this approach would be that the remaining proof obligation:

$$
s_{1} \sqsubseteq_{m} s_{2} \text { implies } \operatorname{Prob}^{\mathcal{D}}\left(s_{1}, \Phi_{1} \mathcal{U}^{\leqslant k} \Phi_{2}\right) \leqslant \operatorname{Prob}^{\mathcal{D}}\left(s_{2}, \Phi_{1} \mathcal{U}^{\leqslant k} \Phi_{2}\right) \text {, for any } k
$$

could be verified by considering the discrete-time behaviour of the CTMC only. Whereas the proof of equation (4) is rather straightforward, the conjecture (5) turns out to be wrong. This is illustrated by the following (uniformised) CTMC $\mathcal{M}$ :

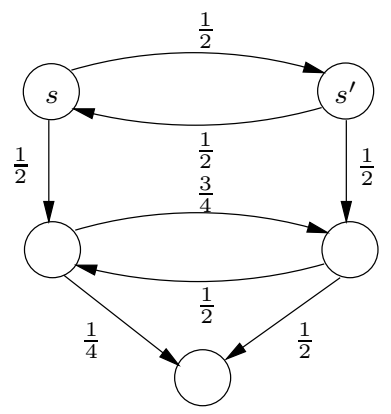


where only the absorbing state is labelled by proposition $b$. It is not difficult to check that state $s^{\prime}$ simulates state $s$. Indeed it follows that $\operatorname{Prob}^{\mathcal{M}}(s, \diamond \leqslant t b) \leqslant$ $\operatorname{Prob}^{\mathcal{M}}\left(s^{\prime}, \diamond \leqslant t b\right)$ for any real time instant $t$. However, $\operatorname{Prob}^{\mathrm{emb}(\mathcal{M})}(s, \diamond \leqslant k b)=$ $\frac{7}{16} \nless \frac{3}{8}=\operatorname{Prob}^{\mathrm{emb}(\mathcal{M})}\left(s^{\prime}, \diamond \leqslant k b\right)$ for $k=3$. This contradicts (5). Thus, this initial proof attempt fails and we have to consider an alternative route. Alternative proof attempts along similar lines failed. We prove (3) therefore in a different way. The crux of the proof is to apply a number of transformations on the CTMC under consideration. The details of the proof are in the appendix; the proof sketch is given below.

Lemma 4. Let $\Phi_{1}$ and $\Phi_{2}$ be CSL-formulas such that the satisfaction sets $\operatorname{Sat}\left(\Phi_{i}\right)$ are upward-closed, i.e., if $s_{1} \sqsubseteq_{m} s_{2}$ and $s_{1} \in \operatorname{Sat}\left(\Phi_{i}\right)$ then $s_{2} \in \operatorname{Sat}\left(\Phi_{i}\right)$ for $i=1,2$. Then:

$$
s_{1} \sqsubseteq m \quad s_{2} \text { implies } \operatorname{Prob}\left(s_{1}, \Phi_{1} \mathcal{U}^{\leqslant t} \Phi_{2}\right) \leqslant \operatorname{Prob}\left(s_{2}, \Phi_{1} \mathcal{U}^{\leqslant t} \Phi_{2}\right) .
$$

Proof. We only provide the proof sketch here; the full proof is given in the appendix. Through a series of transformation steps we modify $\mathcal{M}$ to obtain a CTMC such that for any pair $s_{1} \sqsubseteq m s_{2}$ :

- The probability to move from $s_{1}$ to a $V_{1}$-state equals the probability for the added self-loop $s_{2} \rightarrow s_{2}$.

- The probability for the added self-loop $s_{1} \rightarrow s_{1}$ equals the probability to move from $s_{2}$ to a $V_{2}$-state.

- The probabilities to move from $s_{1}$ and $s_{2}$ to a $U_{1}$ - and $U_{2}$-state, respectively, are equal.

$-s_{2}$ is faster than $s_{1}$, i.e., the exit rate of $s_{2}$ exceeds the exit rate of $s_{1}$.

(The meaning of $U_{1}, U_{2}, V_{1}$ and $V_{2}$ is as in Def. 5.) The reasoning will then be as follows. The interesting case is $s_{i} \models \Phi_{1} \wedge \neg \Phi_{2}$ for $i=1,2$. Hence, all states in $V_{1}$ and $V_{2}$ satisfy $\Phi_{1}$ but not $\Phi_{2}$. Thus, the only possibility for $s_{i}$ to fulfill the path-formula $\Phi_{1} \mathcal{U}^{\leqslant t} \Phi_{2}$ is to move to a $U_{i}$-state. Let $p(s, t, n)$ denote the probability for $s$ to reach a $\Phi_{2}$-state in at most $t$ time units within at most $n$ transitions via $\Phi_{1}$-states. Then, $\operatorname{Prob}\left(s_{i}, \Phi_{1} \mathcal{U} \leqslant t \Phi_{2}\right)$ equals $\lim _{n \rightarrow \infty} p\left(s_{i}, t, n\right)$. Via the introduction of (yet another) state $s_{2}^{\prime}$ that has the same probabilistic behaviour as $s_{2}$ but the exit rate of $s_{1}$ we then establish $p\left(s_{2}^{\prime}, t, n\right) \leqslant p\left(s_{2}, t, n\right)$. By induction on $n$ it is subsequently shown that $p\left(s_{1}, t, n\right) \leqslant p\left(s_{2}^{\prime}, t, n\right)$.

\section{Simulation Equivalence}

This section defines simulation equivalence $\left(\equiv_{m}\right)$ and relates this notion to the equivalences induced by the two CSL fragments. Furthermore, the relationship with lumping equivalence [12], probabilistic (bi)simulation [28,24] and weak probabilistic bisimulation [5] is established. 
Simulation equivalence. Simulation equivalence denotes the kernel of the simulation preorder. Two states are simulation equivalent if and only if they are mutually simulating each other:

Definition 7. $s_{1} \equiv_{m} s_{2}$ if and only if $s_{1} \sqsubseteq_{m} s_{2}$ and $s_{2} \sqsubseteq_{m} s_{1}$.

Theorem 2. Let $s_{1} \equiv_{m} s_{2}$. Then:

1. for any CSL safety-formula $\Phi_{\text {safe }}: s_{1} \models \Phi_{\text {safe }}$ iff $s_{2} \models \Phi_{\text {safe }}$

2. for any CSL liveness-formula $\Phi_{\text {live }}: s_{1} \models \Phi_{\text {live }}$ iff $s_{2} \models \Phi_{\text {live }}$

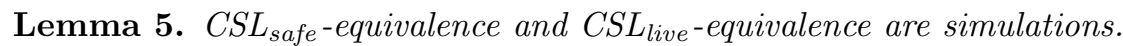

Theorem 3. For any states $s_{1}, s_{2}$ :

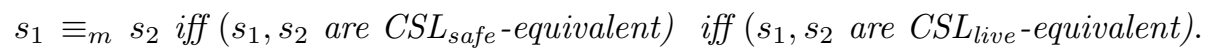

Thus, simulation is characterised by each of the two fragments of CSL we considered.

Lumping equivalence. Recall from [8] that two states $s_{1}$ and $s_{2}$ are lumping equivalent $\left(s_{1} \sim_{m} s_{2}\right)$ if there is some equivalence relation $R$ on $S$ with $\left(s_{1}, s_{2}\right) \in$ $R$ satisfying that whenever $\left(s, s^{\prime}\right) \in R$ then $L(s)=L\left(s^{\prime}\right)$ and for all equivalence classes $C$ in the quotient $S / R$,

$$
\sum_{s^{\prime \prime} \in C} \mathbf{R}\left(s, s^{\prime \prime}\right)=\sum_{s^{\prime \prime} \in C} \mathbf{R}\left(s^{\prime}, s^{\prime \prime}\right)
$$

Theorem 4. For any state $s_{1}, s_{2}: \quad s_{1} \sim_{m} s_{2}$ implies $s_{1} \equiv_{m} s_{2}$.

The converse of the above theorem does not hold. For instance, two corresponding states in a CTMC $\mathcal{M}$ and $\operatorname{unif}(\mathcal{M})$ simulate each other (if considered in the disjoint union of the state spaces), but are not lumping equivalent if the uniformisation rate $E$ is chosen strictly larger than $\max _{s \in S} E(s)$. Thus simulation equivalence strictly refines lumping equivalence.

Simulation on DTMCs. It is interesting to investigate the effect of our simulation relation if interpreted without the constraint on the total rates of states, i.e., on (embedded) DTMCs. For a given DTMC $(S, \mathbf{P}, L)$, let $\leqslant_{p}$ be the preorder obtained by omitting clause 4 . from Def. 5 , and let $\equiv_{p}$ denote the induced simulation equivalence (cf. Def. 7 ). We have that strong probabilistic bisimulation $\left(\sim_{p}\right)$ is finer than $\equiv_{p}$, and so is weak probabilistic bisimulation [5]: Let $\approx_{p}$ denote (state-labelled) weak probabilistic bisimulation. More specific, two states $s_{1}$ and $s_{2}$ are weakly probabilistic bisimilar $\left(s_{1} \approx_{p} s_{2}\right)$ if there is some equivalence relation $R$ on $S$ with $\left(s_{1}, s_{2}\right) \in R$ satisfying that whenever $\left(s, s^{\prime}\right) \in R$ then $L(s)=L\left(s^{\prime}\right)$ and for all equivalence classes $C$ in the quotient $S / R$,

$$
\mathbf{W}(s, C)=\mathbf{W}\left(s^{\prime}, C\right)
$$


where $\mathbf{W}(s, C)=\sum_{s^{\prime \prime} \in[s]_{R}} \mathbf{P}\left(s, s^{\prime \prime}\right) \mathbf{W}\left(s^{\prime \prime}, C\right)$ if $s \notin C$, and 1 otherwise $\left([s]_{R}\right.$ is the equivalence class of $R$ containing $s) .^{3}$

Theorem 5. For any state $s_{1}, s_{2}$ of a DTMC: $s_{1} \approx_{p} s_{2}$ implies $s_{1} \equiv_{p} s_{2}$.

We claim that the converse direction of this theorem holds as well in the DTMC setting (but not for FPSs) though we have not formally shown this yet. Recall that $\sqsubseteq_{p}$ and $\sim_{p}$ agree on DTMCs, and we feel that a similar result may be expected for $\leqslant_{p}$ and $\approx_{p}$. Note that the probabilistic preorder is a side issue of our work since we are mainly interested in CTMC model checking.

\section{Related Work}

Preservation and bisimulation. Aziz et al. [2] have shown that Larsen-Skou probabilistic bisimulation [28] on discrete-time Markov chains fully preserves any formula in the logic Probabilistic CTL (PCTL) [20]. This result has recently been generalised towards continuous-space Markov processes by Desharnais et al. [16]. Segala and Lynch [32] reported similar results for simple probabilistic automata, a model in which probabilistic choices and non-determinism co-exist. Baier et al. [8] have shown that lumping equivalence [12] preserves CSL; Desharnais and Panangaden [17] have recently shown the converse, namely that the equivalence induced by CSL implies lumping equivalence.

Simulation preorders. Based on the seminal works by Larsen and Skou [28] and Jonsson and Larsen [24] on probabilistic (bi)simulation several variants have been proposed, see e.g., $[32,5,7,30,35]$. Mostly related to this paper are the simulations of $[15,32,18]$. We discuss these works briefly.

D'Argenio et al. [15] investigated simulation on discrete-time Markov decision processes, and showed preservation of (untimed) probabilistic reachability properties. Opposed to their work, our approach stays in an entirely probabilistic setting - we do not abstract away probabilistic behaviour. This has the advantage that CSL model-checking algorithms can be applied to the abstract model as well as to the concrete model.

Segala and Lynch [32] presented weak and strong simulations for actionlabelled probabilistic automata and showed that these notions are pre-congruences wrt. parallel composition. For divergence-free probabilistic automata they showed that strong simulation weakly preserves a "safe" fragment of PCTL [20]. In addition, a weak preservation result for weak simulation for a fragment of (a subset of) a variant of PCTL that abstracts from internal activities is shown.

Desharnais et al. [18] studied the approximation of continuous-space Markov processes by a series of finite (rational) Markov processes. They used a simulation preorder to capture the relationship between successive finite approximants and showed that this preorder weakly preserves a subset of PML, a probabilistic variant of Hennessy-Milner logic.

\footnotetext{
${ }^{3}$ Here we define $\approx_{p}$ using the branching bisimulation style, see [5] for a proof that both styles coincide on DTMCs.
} 
Testing preorders. Another important branch of preorders are the ones based on testing, a framework in which processes are compared by their (in)ability to pass a specified set of tests. For discrete-time probabilistic systems, a whole range of testing preorders have been proposed. A recent account can be found in [25] where also the relation between probabilistic may-testing and probabilistic simulation is established. Testing preorders for continuous-time probabilistic systems have received scant attention so far. A notable exception is the work by Bernardo and Cleaveland [10] who consider testing of action-labelled CTMCs. Similar to our simulation preorder, their tests allow one to discriminate models with respect to their transient evolution. To be more precise, two testing preorders are considered, one based on the probability of executing a successful computation whose average duration is not exceeding a time bound, and one based on the probability to reach success within a time bound. It is shown that these testing preorders coincide. CSL preservation results for testing are not known to us.

\section{Concluding Remarks}

This paper presented a simulation preorder $\left(\sqsubseteq_{m}\right)$ for CTMCs and provided weak preservation results for safety- and liveness-fragments of CSL. We claim that the simulation preorder can be easily extended towards Markov reward models (by requiring that rewards of simulating states are related according to $\leqslant$ ) and that weak preservation results for fragments of the logic CSRL [4] can be obtained in a similar way as shown in this paper. As a next step, we plan to work on an algorithm for deciding $\sqsubseteq_{m}$ and to construct the quotient space w.r.t. simulation preorder or simulation equivalence, based on $[5,7,9,30]$. Moreover, we will investigate whether and how the concept of simulation can help to increase the efficiency of CSL model checking using an abstraction refinement methodology as in [15].

\section{A Proofs}

\section{A.1 Basic results for the simulation relation}

Lemma $1 . \sqsubseteq_{m}$ is a preorder.

Proof. Reflexivity follows directly from Def. 5. Transitivity is proven as follows. Let $\sqsubseteq_{1,2}$ and $\sqsubseteq_{2,3}$ be simulations on CTMC $\mathcal{M}$. We show that:

$$
\sqsubseteq=\sqsubseteq_{1,2} \circ \sqsubseteq_{2,3}=\left\{\left(s_{1}, s_{3}\right) \mid \exists s_{2} \in S .\left(s_{1} \sqsubseteq_{1,2} s_{2} \wedge s_{2} \sqsubseteq_{2,3} s_{3}\right)\right\}
$$

is a simulation. Let $s_{1} \sqsubseteq_{1,2} s_{2} \sqsubseteq_{2,3} s_{3}$. It is clear that $s_{1}$ and $s_{3}$ are equally labelled. We check the conditions of Def. 5 for $\sqsubseteq$. Let $U_{i, j}, V_{i, j}, \Delta_{i, j}$ (with $(i, j)=(1,2)$ or $(i, j)=(2,3))$ as in Def. 5. For simplicity, we prove the case where each successor state of $s_{1}$ either belongs to $U_{1,2}$ or to $V_{1,2}$ but not to both, i.e., the function $\delta_{1}$ is the 
characteristic function of $U_{1} \cdot{ }^{4}$ The same condition is assumed for states $s_{2}$ and $s_{3}$. Let $U_{1}=U_{1,2}, V_{1}=V_{1,2}, U_{3}=U_{2,3}$ and $V_{3}=V_{2,3}$. Then:

1. As $\sqsubseteq_{1,2}$ and $\sqsubseteq_{2,3}$ are simulations we have for any state $v_{1} \in V_{1}$ that $v_{1} \sqsubseteq_{1,2} s_{2} \sqsubseteq_{2,3}$ $s_{3}$. Thus, $v_{1} \sqsubseteq s_{3}$. In the same way, we obtain $s_{1} \sqsubseteq v_{3}$ for all $v_{3} \in V_{3}$.

2. Let $U_{2}=U_{1,2} \cap U_{2,3}$ and for $u_{1} \in U_{1}$ and $u_{3} \in U_{3}$ define $\Delta: U_{1} \times U_{3} \rightarrow[0,1]$ by:

$$
\Delta\left(u_{1}, u_{3}\right)=\sum_{u_{2} \in U_{2}} \Delta_{1,2}\left(u_{1}, u_{2}\right) \cdot \Delta_{2,3}\left(u_{2}, u_{3}\right) \cdot \frac{K_{2}}{\mathbf{P}\left(s_{2}, u_{2}\right)}
$$

If $\Delta\left(u_{1}, u_{3}\right)>0$ then there exists some $u_{2} \in U_{2}$ with $\Delta_{1,2}\left(u_{1}, u_{2}\right)>0$ and $\Delta_{2,3}\left(u_{2}, u_{3}\right)>0$. Hence, $u_{1} \sqsubseteq_{1,2} u_{2} \sqsubseteq_{2,3} u_{3}$, and by definition of $u_{1} \sqsubseteq u_{3}$.

3. Let $K_{i}=\sum_{u_{i} \in U_{i}} \mathbf{P}\left(s_{i}, u_{i}\right)$, for $i=1,2,3$. Then, using the above definition of $\Delta$ we derive:

$$
\begin{aligned}
K_{3} \cdot \sum_{u_{1} \in U_{1}} \Delta\left(u_{1}, u_{3}\right) & =K_{3} \cdot \sum_{u_{1} \in U_{1}} \sum_{u_{2} \in U_{2}} \Delta_{1,2}\left(u_{1}, u_{2}\right) \cdot \Delta_{2,3}\left(u_{2}, u_{3}\right) \cdot \frac{K_{2}}{\mathbf{P}\left(s_{2}, u_{2}\right)} \\
& =K_{3} \cdot \sum_{u_{2} \in U_{2}} \Delta_{2,3}\left(u_{2}, u_{3}\right) \cdot \frac{K_{2}}{\mathbf{P}\left(s_{2}, u_{2}\right)} \cdot \sum_{u_{1} \in U_{1}} \Delta_{1,2}\left(u_{1}, u_{2}\right) \\
& =K_{3} \cdot \sum_{u_{2} \in U_{2}} \Delta_{2,3}\left(u_{2}, u_{3}\right) \cdot \frac{1}{\mathbf{P}\left(s_{2}, u_{2}\right)} \cdot \mathbf{P}\left(s_{2}, u_{2}\right) \\
& =K_{3} \cdot \sum_{u_{2} \in U_{2}} \Delta_{2,3}\left(u_{2}, u_{3}\right)=\mathbf{P}\left(s_{3}, u_{3}\right) .
\end{aligned}
$$

Similarly, we get $K_{1} \cdot \sum_{u_{3} \in U_{3}} \Delta\left(u_{1}, u_{3}\right)=\mathbf{P}\left(s_{1}, u_{1}\right)$.

4. Follows from: $K_{1} \cdot E\left(s_{1}\right) \stackrel{\leqslant}{\leqslant} K_{2} \cdot E\left(s_{2}\right) \leqslant K_{3} \cdot E\left(s_{3}\right)$.

Lemma 2. For CTMC $\mathcal{M}=(S, \mathbf{R}, L)$ and $s_{1}, s_{2} \in S$ we have:

$$
s_{1} \sqsubseteq_{m}^{\mathcal{M}} s_{2} \text { if and only if } s_{1} \sqsubseteq_{m}^{\operatorname{unif}(\mathcal{M})} s_{2} \text {. }
$$

Proof. Let $s_{1} \sqsubseteq \stackrel{\mathcal{M}}{\mathcal{M}} s_{2}$ and let $\sqsubseteq, \delta_{i}, U_{i}, V_{i}, K_{i}$ (for $i=1,2$ ) and $\Delta$ as in Def. 5 . The same components $U_{i}, V_{i}$ and $\Delta$ can be used to show that $\sqsubseteq$ is a simulation on unif $(\mathcal{M})=$ $(S, \overline{\mathbf{R}}, L)$. Let $\overline{\delta_{1}}(s)=\delta_{1}(s)$ if $s \neq s_{1}$ and $\overline{\delta_{1}}\left(s_{1}\right)=\delta_{1}\left(s_{1}\right) \cdot \mathbf{R}\left(s_{1}, s_{1}\right) / \overline{\mathbf{R}}\left(s_{1}, s_{1}\right)$, and $\overline{\delta_{2}}$ be defined similarly. We show that $\sqsubseteq_{m}$ is a simulation on unif $(\mathcal{M})$ by checking the conditions of Def. 5. It suffices to check constraints 3 and 4 ; the other constraints are clear. We have:

$$
\begin{aligned}
& \text { 3. Let } \overline{\overline{K_{i}}}=\sum_{u_{i} \in U_{i}} \overline{\delta_{i}}\left(u_{i}\right) \cdot \overline{\mathbf{P}}\left(s_{i}, u_{i}\right) \text {. For } u_{1} \in U_{1} \backslash\left\{s_{1}\right\}: \\
& \qquad \begin{aligned}
\overline{K_{1}} \cdot \sum_{u_{2} \in U_{2}} \Delta\left(u_{1}, u_{2}\right) & =\frac{E\left(s_{1}\right)}{E} \cdot K_{1} \cdot \sum_{u_{2} \in U_{2}} \Delta\left(u_{1}, u_{2}\right) \\
& =\frac{E\left(s_{1}\right)}{E} \cdot \delta_{1}\left(u_{1}\right) \cdot \mathbf{P}\left(s_{1}, u_{1}\right) \\
& =\delta_{1}\left(u_{1}\right) \cdot \frac{\mathbf{R}\left(s_{1}, u_{1}\right)}{E}=\overline{\delta_{1}}\left(u_{1}\right) \cdot \overline{\mathbf{P}}\left(s_{1}, u_{1}\right),
\end{aligned}
\end{aligned}
$$

\footnotetext{
${ }^{4}$ The justification for this simplification is as follows. For the proof of the general case we have to replace any occurrence of $\mathbf{P}\left(s_{1}, u_{1}\right)$ (for $\left.u_{1} \in U_{1}\right)$ by $\delta_{1}\left(u_{1}\right) \cdot \mathbf{P}\left(s_{1}, u_{1}\right)$ and $\mathbf{P}\left(s_{1}, v_{1}\right)$ (for $\left.v_{1} \in V_{1}\right)$ by $\left(1-\delta_{1}\left(v_{1}\right)\right) \cdot \mathbf{P}\left(s_{1}, v_{1}\right)$.
} 
where $\overline{\mathbf{P}}\left(s_{i}, u_{i}\right)=\overline{\mathbf{R}}\left(s_{i}, u_{i}\right) / E$ are the transition probabilities from state $s_{i}$ in unif $(\mathcal{M})$. For $s_{1} \in U_{1}$ we have: $\overline{K_{1}} \cdot \sum_{u_{2} \in U_{2}} \Delta\left(s_{1}, u_{2}\right)=\delta_{1}\left(s_{1}\right) \cdot \frac{\mathbf{R}\left(s_{1}, s_{1}\right)}{E}$. Since:

$$
\overline{\delta_{1}}\left(s_{1}\right) \cdot \overline{\mathbf{P}}\left(s_{1}, s_{1}\right)=\frac{\mathbf{R}\left(s_{1}, s_{1}\right)}{\overline{\mathbf{R}}\left(s_{1}, s_{1}\right)} \cdot \delta_{1}\left(s_{1}\right) \cdot \frac{\overline{\mathbf{R}}\left(s_{1}, s_{1}\right)}{E}=\delta_{1}\left(s_{1}\right) \cdot \frac{\mathbf{R}\left(s_{1}, s_{1}\right)}{E},
$$

it follows

$$
\overline{K_{1}} \cdot \sum_{u_{2} \in U_{2}} \Delta\left(s_{1}, u_{2}\right)=\overline{\delta_{1}}\left(s_{1}\right) \cdot \overline{\mathbf{P}}\left(s_{1}, s_{1}\right) .
$$

In the same way, condition 3. can be proven for state $s_{2}$.

4. Then:

$$
\begin{aligned}
\overline{K_{1}} \cdot E & =\sum_{u_{1} \in U_{1}} \overline{\delta_{1}}\left(u_{1}\right) \cdot \overline{\mathbf{R}}\left(s_{1}, u_{1}\right) \\
& =\sum_{\substack{u_{1} \in U_{1} \\
u_{1} \neq s_{1}}} \delta_{1}\left(u_{1}\right) \cdot \mathbf{R}\left(s_{1}, u_{1}\right)+\delta_{1}\left(s_{1}\right) \cdot \frac{\mathbf{R}\left(s_{1}, s_{1}\right)}{\overline{\mathbf{R}}\left(s_{1}, s_{1}\right)} \cdot \overline{\mathbf{R}}\left(s_{1}, s_{1}\right) \\
& =\sum_{\substack{u_{1} \in U_{1} \\
u_{1} \neq s_{1}}} \delta_{1}\left(u_{1}\right) \cdot \mathbf{R}\left(s_{1}, u_{1}\right)+\delta_{1}\left(s_{1}\right) \cdot \mathbf{R}\left(s_{1}, s_{1}\right) \\
& =\sum_{u_{1} \in U_{1}} \mathbf{R}\left(s_{1}, u_{1}\right)=K_{1} \cdot E\left(s_{1}\right)
\end{aligned}
$$

By a similar argument it follows that $\overline{K_{2}} \cdot E=K_{2} \cdot E\left(s_{2}\right)$. Since $K_{1} \cdot E\left(s_{1}\right) \leqslant$ $K_{2} \cdot E\left(s_{2}\right)$ we thus have $\overline{K_{1}} \cdot E \leqslant \overline{K_{2}} \cdot E$.

\section{A.2 Proving weak preservation}

From Lemma 3, it follows that the values $\operatorname{Prob}\left(s, \Phi_{1} \mathcal{U} \leqslant t \Phi_{2}\right)$ remain unaffected when switching from $\mathcal{M}$ to its uniformized CTMC. This allows us to assume w.l.o.g. that all states in $\mathcal{M}$ have the same exit rate: $E=E(s)$ for all states $s$. For the sake of simplicity we assume $E=1$ in the sequel. We first present two transformations on CTMCs that are shown to preserve probabilities for time-bounded until-formulas.

First transformation. We replace $\mathcal{M}$ by a lumping equivalent uniformized CTMC $\mathcal{M}^{\prime}$. The states of this transformed CTMC are of the form $\left\langle s_{1}, s_{2}, i\right\rangle$ with $i=$ 1,2 . Intuitively, $\left\langle s_{1}, s_{2}, 1\right\rangle$ and $\left\langle s_{1}, s_{2}, 2\right\rangle$ are copies of states $s_{1}$ and $s_{2}$, respectively. For any pair $\left\langle s_{1}, s_{2}\right\rangle$ of states in $\mathcal{M}$ with $s_{1} \sqsubseteq_{m} s_{2}$ we fix $\delta_{1}=\delta_{1}^{\left\langle s_{1}, s_{2}\right\rangle}$, $\delta_{2}=\delta_{2}^{\left\langle s_{1}, s_{2}\right\rangle}$ and a weight function $\Delta=\Delta^{\left\langle s_{1}, s_{2}\right\rangle}$ as in Def. 5. Furthermore, $U_{1}$, $U_{2}, V_{1}, V_{2}, K_{1}, K_{2}$ are as in Def. 5 . In particular, we have: $K_{1} \leqslant K_{2}$ because $\mathcal{M}$ is uniformized. ${ }^{5}$

\footnotetext{
${ }^{5}$ The sets $U_{1}, U_{2}, V_{1}, V_{2}$ as well as $K_{1}, K_{2}$ depend on $\left\langle s_{1}, s_{2}\right\rangle$. Thus, it would be more precise to write $U_{1}^{\left\langle s_{1}, s_{2}\right\rangle}, U_{2}^{\left\langle s_{1}, s_{2}\right\rangle}$, etc. For simplicity, we omit these parameters.
} 
Definition 8. For CTMC $\mathcal{M}=(S, \mathbf{R}, L)$ let CTMC $\mathcal{M}^{\prime}=\left(S^{\prime}, \mathbf{R}^{\prime}, L^{\prime}\right)$ be defined by $S^{\prime}=\left\{\left\langle s_{1}, s_{2}, i\right\rangle \mid s_{1} \sqsubseteq_{m} s_{2} \wedge i=1,2\right\}$ and $L^{\prime}\left(\left\langle s_{1}, s_{2}, i\right\rangle\right)=L\left(s_{i}\right)$ for $i=1,2$, and the rate matrix:

$\mathbf{R}^{\prime}\left(\left\langle s_{1}, s_{2}, 1\right\rangle, w\right)= \begin{cases}K_{1} \cdot \delta_{1}\left(u_{1}\right) \cdot \Delta\left(u_{1}, u_{2}\right) & \text { if } w=\left\langle u_{1}, u_{2}, 1\right\rangle \text { and } \Delta\left(u_{1}, u_{2}\right)>0 \\ \left(1-\delta_{1}\left(v_{1}\right)\right) \cdot \mathbf{P}\left(s_{1}, v_{1}\right) & \text { if } w=\left\langle v_{1}, s_{2}, 1\right\rangle \text { and } \delta_{1}\left(v_{1}\right)<1 \\ 0 & \text { otherwise, }\end{cases}$

and

$\mathbf{R}^{\prime}\left(\left\langle s_{1}, s_{2}, 2\right\rangle, w\right)= \begin{cases}K_{2} \cdot \delta_{2}\left(u_{2}\right) \cdot \Delta\left(u_{1}, u_{2}\right) & \text { if } w=\left\langle u_{1}, u_{2}, 2\right\rangle \text { and } \Delta\left(u_{1}, u_{2}\right)>0 \\ \left(1-\delta_{2}\left(v_{2}\right)\right) \cdot \mathbf{P}\left(s_{2}, v_{2}\right) & \text { if } w=\left\langle s_{1}, v_{2}, 2\right\rangle \text { and } \delta_{2}\left(v_{2}\right)<1 \\ 0 & \text { otherwise. }\end{cases}$

In the sequel, let $\mathcal{M}^{\prime}$ be constructed according to Def. 8. Let lumping equivalence (denoted $\sim_{m}$ ) be defined as in Section 5 . It follows by construction that for $i=1,2$ :

Proposition 1. $s_{i} \sim_{m}\left\langle s_{1}, s_{2}, i\right\rangle$.

By the results of $[8]$ it now directly follows that this transformation leaves the probabilities for time-bounded until-formulas invariant:

Corollary 1. $\operatorname{Prob}^{\mathcal{M}}\left(s_{i}, \Phi_{1} \mathcal{U}^{\leqslant t} \Phi_{2}\right)=\operatorname{Prob}^{\mathcal{M}^{\prime}}\left(\left\langle s_{1}, s_{2}, i\right\rangle, \Phi_{1} \mathcal{U}^{\leqslant t} \Phi_{2}\right)$.

Second transformation. To simplify the following exposition, we assume that:

- $U_{1} \cap V_{1}=\varnothing$ and $U_{2} \cap V_{2}=\varnothing$; otherwise, we work with further copies of the states that belong to both $U_{i}$ and $V_{i}$.

- $s_{1} \notin U_{1} \cup V_{1}$ and $s_{2} \notin U_{2} \cup V_{2}$; again, if these conditions are not fulfilled we may deal with additional copies of states $s_{1}$ and $s_{2}$ if they belong to $U_{i}$ or $V_{i}$ (i.e., if $s_{i}$ has a self-loop in $\mathcal{M}$ ).

We now modify $\mathcal{M}^{\prime}$ - constructed as in Def. 8 - by adding transitions as follows. Adding a self-loop (with arbitrary rate) does not change the transient probabil-

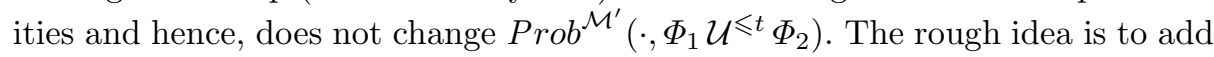
a self-loop to state $\left\langle s_{1}, s_{2}, 1\right\rangle$ with rate

$$
\lambda= \begin{cases}\left(\frac{1}{K_{2}}-1\right) \cdot K_{1}: & \text { if } K_{1} \neq 0 \\ 0 & : \text { otherwise }\end{cases}
$$

while $\left\langle s_{1}, s_{2}, 2\right\rangle$ is extended by a self-loop with rate

$$
\mu= \begin{cases}\left(\frac{1}{K_{1}}-1\right) \cdot K_{2}: & \text { if } K_{1} \neq 0 \\ 0 & : \text { otherwise. }\end{cases}
$$

Instead of adding a self-loop, we insert transitions from $\left\langle s_{1}, s_{2}, 1\right\rangle$ to the copies $\left\langle s_{1}, v_{2}, 1\right\rangle$ of state $s_{1}$ where $v_{2}$ ranges over all elements in $V_{2}$, such that the rates of these auxiliary transitions sum up to $\lambda$. We do the same with state $\left\langle s_{1}, s_{2}, 2\right\rangle$ for which we insert transitions to $\left\langle v_{1}, s_{2}, 2\right\rangle, v_{1} \in V_{1}$, with total rate $\mu$. 
Definition 9. For CTMC $\mathcal{M}^{\prime}=\left(S^{\prime}, \mathbf{R}^{\prime}, L^{\prime}\right)$ as defined in Def. 8 as the transformed version of CTMC $\mathcal{M}$ and $\lambda$ and $\mu$ as defined before, the CTMC $\mathcal{M}^{\prime \prime}=$ $\left(S^{\prime}, \mathbf{R}^{\prime \prime}, L^{\prime}\right)$ is defined by: ${ }^{6}$

$$
\begin{aligned}
\mathbf{R}^{\prime \prime}\left(\left\langle s_{1}, s_{2}, 1\right\rangle,\left\langle s_{1}, v_{2}, 1\right\rangle\right) & =\lambda \cdot \frac{\mathbf{P}\left(s_{2}, v_{2}\right)}{1-K_{2}}, \text { for } v_{1} \in V_{1} \\
\mathbf{R}^{\prime \prime}\left(\left\langle s_{1}, s_{2}, 2\right\rangle,\left\langle v_{1}, s_{2}, 2\right\rangle\right) & =\mu \cdot \frac{\mathbf{P}\left(s_{1}, v_{1}\right)}{1-K_{1}}, \text { for } v_{2} \in V_{2} \\
\mathbf{R}^{\prime \prime}\left(\left\langle s_{1}, s_{2}, i\right\rangle, w\right) & =\mathbf{R}^{\prime}\left(\left\langle s_{1}, s_{2}, i\right\rangle, w\right) \text { for all other states } w .
\end{aligned}
$$

Note that $\mathcal{M}^{\prime \prime}$ and $\mathcal{M}^{\prime}$ have the same state space and the same labellings; they only differ in the rate matrix. As we assume $U_{i} \cap V_{i}=\varnothing$ we now have:

$$
\begin{aligned}
\mathbf{R}^{\prime \prime}\left(\left\langle s_{1}, s_{2}, 2\right\rangle,\left\langle u_{1}, u_{2}, 2\right\rangle\right) & =K_{2} \cdot \Delta\left(u_{1}, u_{2}\right) \\
\mathbf{R}^{\prime \prime}\left(\left\langle s_{1}, s_{2}, 2\right\rangle,\left\langle s_{1}, v_{2}, 2\right\rangle\right) & =\mathbf{P}\left(s_{2}, v_{2}\right) \\
\mathbf{R}^{\prime \prime}\left(\left\langle s_{1}, s_{2}, 1\right\rangle,\left\langle u_{1}, u_{2}, 1\right\rangle\right) & =K_{1} \cdot \Delta\left(u_{1}, u_{2}\right) \\
\mathbf{R}^{\prime \prime}\left(\left\langle s_{1}, s_{2}, 1\right\rangle,\left\langle v_{1}, s_{2}, 1\right\rangle\right) & =\mathbf{P}\left(s_{1}, v_{1}\right)
\end{aligned}
$$

As all states $\left\langle s_{1}, s_{2}, 1\right\rangle,\left\langle s_{1}, v_{2}, 1\right\rangle$ fall in the same lumping equivalence class, we have - again using the results of [8] - for $i=1,2$ :

Proposition 2. $\operatorname{Prob}^{\mathcal{M}^{\prime}}\left(\left\langle s_{1}, s_{2}, i\right\rangle, \Phi_{1} \mathcal{U} \leqslant t \Phi_{2}\right)=\operatorname{Prob}^{\mathcal{M}^{\prime \prime}}\left(\left\langle s_{1}, s_{2}, i\right\rangle, \Phi_{1} \mathcal{U} \leqslant t \Phi_{2}\right)$.

Thus, also the second transformation does not affect the probabilities for timebounded until-formulas. Before we continue we briefly justify the construction of $\mathcal{M}^{\prime \prime}$. The total exit-rates of states $\left\langle s_{1}, s_{2}, 1\right\rangle$ and $\left\langle s_{1}, s_{2}, 2\right\rangle$ in $\mathcal{M}^{\prime \prime}$ are: ${ }^{7}$

$$
E^{\prime \prime}\left(\left\langle s_{1}, s_{2}, 1\right\rangle\right)=1+\lambda \leqslant 1+\mu=E^{\prime \prime}\left(\left\langle s_{1}, s_{2}, 2\right\rangle\right)
$$

Note that $K_{1} \leqslant K_{2}$ and hence, $1 / K_{2} \leqslant 1 / K_{1}$, if $K_{1}>0$. Hence:

$$
\lambda=\left(\frac{1}{K_{2}}-1\right) \cdot K_{1} \leqslant\left(\frac{1}{K_{1}}-1\right) \cdot K_{2}=\mu
$$

If $K_{1}=0$ then $\lambda=\mu=0$.

The transition probabilities are as indicated in the informal proof sketch. The total probability for $\left\langle s_{1}, s_{2}, 1\right\rangle$ to move to state $\left\langle v_{1}, s_{2}, 1\right\rangle$ is

$$
\begin{aligned}
& \mathbf{P}^{\prime \prime}\left(\left\langle s_{1}, s_{2}, 1\right\rangle,\left\langle v_{1}, s_{2}, 1\right\rangle\right)=\frac{\mathbf{P}\left(s_{1}, v_{1}\right)}{1+\lambda}=\frac{\mathbf{P}\left(s_{1}, v_{1}\right)}{1+\left(1 / K_{2}-1\right) K_{1}} \\
& =\frac{K_{2} \cdot \mathbf{P}\left(s_{1}, v_{1}\right)}{K_{2}+\left(1-K_{2}\right) K_{1}}=\frac{K_{2} \cdot \mathbf{P}\left(s_{1}, v_{1}\right)}{K_{2}+K_{1}-K_{2} \cdot K_{1}} .
\end{aligned}
$$

\footnotetext{
${ }^{6}$ Here, we assume $K_{1}<1$ and $K_{2}<1$. If $K_{1}=1$, we have $V_{1}=\varnothing$ and we do not insert auxiliary transitions. The same applies when $K_{2}=1$.

${ }^{7}$ Recall that all states in $\mathcal{M}$ have the total rate $E=1$.
} 
This equals the probability for the auxiliary transition from $\left\langle s_{1}, s_{2}, 2\right\rangle$ to $\left\langle v_{1}, s_{2}, 2\right\rangle$ as we have (for $0<K_{1}<1$ ):

$$
\begin{aligned}
& \mathbf{P}^{\prime \prime}\left(\left\langle s_{1}, s_{2}, 2\right\rangle,\left\langle v_{1}, s_{2}, 2\right\rangle\right)=\frac{\mathbf{P}\left(s_{1}, v_{1}\right)}{1-K_{1}} \cdot \frac{\mu}{1+\mu}= \\
& \frac{\mathbf{P}\left(s_{1}, v_{1}\right)}{1-K_{1}} \cdot \frac{\left(1-K_{1}\right) K_{2}}{K_{1}+\left(1-K_{1}\right) K_{2}}=\frac{\mathbf{P}\left(s_{1}, v_{1}\right) \cdot K_{2}}{K_{1}+\left(1-K_{1}\right) K_{2}}=\frac{K_{2} \cdot \mathbf{P}\left(s_{1}, v_{1}\right)}{K_{1}+K_{2}-K_{1} \cdot K_{2}}
\end{aligned}
$$

Similarly, the probability for the auxiliary transition from $\left\langle s_{1}, s_{2}, 1\right\rangle$ to $\left\langle s_{1}, v_{2}, 1\right\rangle$ coincides with the probability for $\left\langle s_{1}, s_{2}, 2\right\rangle$ to move to state $\left\langle s_{1}, v_{2}, 2\right\rangle$. Thus:

$$
\begin{aligned}
& \mathbf{P}^{\prime \prime}\left(\left\langle s_{1}, s_{2}, 1\right\rangle,\left\langle v_{1}, s_{2}, 1\right\rangle\right)=\mathbf{P}^{\prime \prime}\left(\left\langle s_{1}, s_{2}, 2\right\rangle,\left\langle v_{1}, s_{2}, 2\right\rangle\right) \\
& \mathbf{P}^{\prime \prime}\left(\left\langle s_{1}, s_{2}, 1\right\rangle,\left\langle s_{1}, v_{2}, 1\right\rangle\right)=\mathbf{P}^{\prime \prime}\left(\left\langle s_{1}, s_{2}, 2\right\rangle,\left\langle s_{1}, v_{2}, 2\right\rangle\right)
\end{aligned}
$$

The probability for $\left\langle s_{1}, s_{2}, 1\right\rangle$ to move to state $\left\langle u_{1}, u_{2}, 1\right\rangle$ (where $u_{i} \in U_{i}$ ) is (provided $0<K_{2}<1$ ):

$$
\begin{aligned}
& \mathbf{P}^{\prime \prime}\left(\left\langle s_{1}, s_{2}, 1\right\rangle,\left\langle u_{1}, u_{2}, 1\right\rangle\right)=\frac{K_{1} \cdot \Delta\left(u_{1}, u_{2}\right)}{1+\lambda}=\frac{K_{1} \cdot \Delta\left(u_{1}, u_{2}\right)}{1+\left(1 / K_{2}-1\right) K_{1}} \\
& =\frac{K_{1} \cdot K_{2} \cdot \Delta\left(u_{1}, u_{2}\right)}{K_{2}+\left(1-K_{2}\right) K_{1}}=\frac{K_{1} \cdot K_{2} \cdot \Delta\left(u_{1}, u_{2}\right)}{K_{2}+K_{1}-K_{2} \cdot K_{1}}
\end{aligned}
$$

The probability for $\left\langle s_{1}, s_{2}, 2\right\rangle$ to go to state $\left\langle u_{1}, u_{2}, 2\right\rangle$ is (provided $0<K_{1}<1$ ):

$$
\begin{aligned}
& \mathbf{P}^{\prime \prime}\left(\left\langle s_{1}, s_{2}, 2\right\rangle,\left\langle u_{1}, u_{2}, 2\right\rangle\right)=\frac{K_{2} \cdot \Delta\left(u_{1}, u_{2}\right)}{1+\mu}=\frac{K_{2} \cdot \Delta\left(u_{1}, u_{2}\right)}{1+\left(1 / K_{1}-1\right) K_{2}} \\
& =\frac{K_{1} \cdot K_{2} \cdot \Delta\left(u_{1}, u_{2}\right)}{K_{1}+\left(1-K_{1}\right) K_{2}}=\frac{K_{1} \cdot K_{2} \cdot \Delta\left(u_{1}, u_{2}\right)}{K_{1}+K_{2}-K_{1} \cdot K_{2}}
\end{aligned}
$$

Hence, we have:

$$
\mathbf{P}^{\prime \prime}\left(\left\langle s_{1}, s_{2}, 1\right\rangle,\left\langle u_{1}, u_{2}, 1\right\rangle\right)=\mathbf{P}^{\prime \prime}\left(\left\langle s_{1}, s_{2}, 2\right\rangle,\left\langle u_{1}, u_{2}, 2\right\rangle\right)
$$

If $K_{1}=0$ or $K_{2}=0$ we have $K_{1}=K_{2}=0$ and $U_{1}=U_{2}=\varnothing$ (because of the "weight-function condition") and $\lambda=\mu=0$. If $K_{1}=1$ or $K_{2}=1$ then $\lambda=\mu=0$. In either case the above equations for the transition probabilities also hold.

Definition 10. Let $p(s, t, n)$ be the probability to reach a $\Phi_{2}$-state via $\Phi_{1}$-states within $n(n \geqslant 0)$ steps and time-bound $t$ from state $s$. This function is defined by:

$$
\begin{aligned}
p(s, t, n) & =1 \text { if } s \models \Phi_{2} \\
p(s, t, n) & =0 \text { if } s \not \models\left(\Phi_{1} \vee \Phi_{2}\right) \text { or }\left(n=0 \text { and } s \not \models \Phi_{2}\right) \\
p(s, t, n+1) & =\sum_{s^{\prime}} \mathbf{R}\left(s, s^{\prime}\right) \cdot \int_{0}^{t} e^{-E(s) \cdot x} \cdot p(s, t-x, n) d x .
\end{aligned}
$$


The last defining clause is informally justified as follows. If $s$ satisfies $\Phi_{1}$ and $\neg \Phi_{2}$, the probability of reaching a $\Phi_{2}$-state from $s$ within $t$ time units and $n+1$ steps equals the probability of reaching some direct successor $s^{\prime}$ of $s$ in $x$ time units $(x \leqslant t)$, multiplied by the probability of reaching a $\Phi_{2}$-state from $s^{\prime}$ in the remaining time $t-x$ (along a $\Phi_{1}$-path) in $n$ steps.

Lemma 6. Let $\mathcal{M}$ be a CTMC and $\mathcal{M}^{\prime \prime}$ be defined according to Def. 9 and let $\operatorname{Sat}\left(\Phi_{i}\right)$ be upward-closed, i.e., if $s_{1} \sqsubseteq_{m} s_{2}$ and $s_{1} \in \operatorname{Sat}\left(\Phi_{i}\right)$ then $s_{2} \in \operatorname{Sat}\left(\Phi_{i}\right)$ for $i=1,2$. Then for any states $s_{1}, s_{2}$ such that $s_{1} \sqsubseteq_{m} s_{2}$ and any non-negative real $t$ we have for any $n \geqslant 0$ :

$$
p\left(\left\langle s_{1}, s_{2}, 1\right\rangle, t, n\right) \leqslant p\left(\left\langle s_{1}, s_{2}, 2\right\rangle, t, n\right) .
$$

Proof. The claim is clear (for all $n \geqslant 0$ ) if $s_{2}$ is a $\Phi_{2}$-state because then

$$
\operatorname{Prob}^{\mathcal{M}^{\prime \prime}}\left(\left\langle s_{1}, s_{2}, 1\right\rangle, t, n\right) \leqslant 1=\operatorname{Prob}^{\mathcal{M}^{\prime \prime}}\left(\left\langle s_{1}, s_{2}, 2\right\rangle, t, n\right) .
$$

(Recall that $s_{i}$ and $\left\langle s_{1}, s_{2}, i\right\rangle$ are lumping equivalent. Hence, they satisfy the same CSL-formulas.) Similarly, if $s_{1} \models \neg \Phi_{1} \wedge \neg \Phi_{2}$ then

$$
\operatorname{Prob}^{\mathcal{M}^{\prime \prime}}\left(\left\langle s_{1}, s_{2}, 1\right\rangle, t, n\right)=0 \leqslant \operatorname{Prob}^{\mathcal{M}^{\prime \prime}}\left(\left\langle s_{1}, s_{2}, 2\right\rangle, t, n\right) .
$$

The proof of the remaining case $s_{1}, s_{2} \models \Phi_{1} \wedge \neg \Phi_{2}$ is by induction on $n .^{8}$

The basis of induction is clear as we have

$$
p\left(\left\langle s_{1}, s_{2}, 1\right\rangle, t, 0\right)=0=p\left(\left\langle s_{1}, s_{2}, 2\right\rangle, t, 0\right)
$$

Induction step $n \Longrightarrow n+1$ : We extend $\mathcal{M}^{\prime \prime}$ (yet again) by a copy of the state $\left\langle s_{1}, s_{2}, 2\right\rangle$. Let $\left\langle s_{1}, s_{2}, 2\right.$, slow $\rangle$ be a new state in $\mathcal{M}^{\prime \prime}$ which has the same successors as the states $\left\langle s_{1}, s_{2}, 2\right\rangle$ (with the same transition probabilities) but the total rate of $\left\langle s_{1}, s_{2}, 1\right\rangle$. That is:

$$
\mathbf{P}^{\prime \prime}\left(\left\langle s_{1}, s_{2}, 2, \text { slow }\right\rangle, w\right)=\mathbf{P}^{\prime \prime}\left(\left\langle s_{1}, s_{2}, 2\right\rangle, w\right)
$$

for all states $w$ in $\mathcal{M}^{\prime \prime}$ and

$$
E^{\prime \prime}\left(\left\langle s_{1}, s_{2}, 2, \text { slow }\right\rangle\right)=E^{\prime \prime}\left(\left\langle s_{1}, s_{2}, 1\right\rangle\right)=1+\lambda
$$

(The auxiliary state $\left\langle s_{1}, s_{2}, 2\right.$, slow $\rangle$ does not have any predecessors.) As state $\left\langle s_{1}, s_{2}, 2\right.$, slow $\rangle$ is slower than $\left\langle s_{1}, s_{2}, 2\right\rangle$ (but has the same transition probabilities) we have:

$$
p\left(\left\langle s_{1}, s_{2}, 2, \text { slow }\right\rangle, t, n+1\right) \leqslant p\left(\left\langle s_{1}, s_{2}, 2\right\rangle, t, n+1\right)
$$

The induction hypothesis yields that

$$
\begin{aligned}
& p\left(\left\langle s_{1}, v_{2}, 1\right\rangle, y, n\right) \leqslant p\left(\left\langle s_{1}, v_{2}, 2\right\rangle, y, n\right) \\
& p\left(\left\langle v_{1}, s_{2}, 1\right\rangle, y, n\right) \leqslant p\left(\left\langle v_{1}, s_{2}, 2\right\rangle, y, n\right) \\
& p\left(\left\langle u_{1}, u_{2}, 1\right\rangle, y, n\right) \leqslant p\left(\left\langle u_{1}, u_{2}, 2\right\rangle, y, n\right)
\end{aligned}
$$

\footnotetext{
${ }^{8}$ Note that $\operatorname{Sat}\left(\Phi_{i}\right)$ is upward-closed, hence the cases $\left(s_{1} \models \Phi_{2}\right) \wedge\left(s_{2} \not \models \Phi_{2}\right)$ and $\left(s_{1} \models \Phi_{1} \wedge \neg \Phi_{2}\right) \wedge\left(s_{2} \models \neg \Phi_{1} \wedge \neg \Phi_{2}\right)$ are impossible.
} 
for any real number $y$ and states $v_{1} \in V_{1}, v_{2} \in V_{2}$ and all states $u_{1} \in U_{1}, u_{2} \in U_{2}$ where $\Delta\left(u_{1}, u_{2}\right)>0$. Hence, we get:

$$
\begin{aligned}
& p\left(\left\langle s_{1}, s_{2}, 2\right\rangle, t, n+1\right) \\
& \geqslant p\left(\left\langle s_{1}, s_{2}, 2, \text { slow }\right\rangle, t, n+1\right) \\
& =\sum_{w}(1+\lambda) \cdot \mathbf{P}^{\prime \prime}\left(\left\langle s_{1}, s_{2}, 2\right\rangle, w\right) \cdot \int_{0}^{t} e^{-(1+\lambda) x} \cdot p(w, t-x, n) d x \\
& =\sum_{v_{1} \in V_{1}}(1+\lambda) \cdot \underbrace{\mathbf{P}^{\prime \prime}\left(\left\langle s_{1}, s_{2}, 2\right\rangle,\left\langle v_{1}, s_{2}, 2\right\rangle\right)}_{=\mathbf{P}^{\prime \prime}\left(\left\langle s_{1}, s_{2}, 1\right\rangle,\left\langle v_{1}, s_{2}, 1\right\rangle\right)} \cdot \int_{0}^{t} e^{-(1+\lambda) x} \cdot \underbrace{p\left(\left\langle v_{1}, s_{2}, 2\right\rangle, t-x, n\right)}_{\geqslant p\left(\left\langle v_{1}, s_{2}, 1\right\rangle, t-x, n\right)} d x \\
& +\sum_{v_{2} \in V_{2}}(1+\lambda) \cdot \underbrace{\mathbf{P}^{\prime \prime}\left(\left\langle s_{1}, s_{2}, 2\right\rangle,\left\langle s_{1}, v_{2}, 2\right\rangle\right)}_{=\mathbf{P}^{\prime \prime}\left(\left\langle s_{1}, s_{2}, 1\right\rangle,\left\langle s_{1}, v_{2}, 1\right\rangle\right)} \cdot \int_{0}^{t} e^{-(1+\lambda) x} \cdot \underbrace{p\left(\left\langle s_{1}, v_{2}, 2\right\rangle, t-x, n\right)}_{\geqslant p\left(\left\langle s_{1}, v_{2}, 1\right\rangle, t-x, n\right)} d x \\
& +\sum_{\substack{u_{2} \in U_{2} \\
u_{1} \in U_{1}}}(1+\lambda) \cdot \underbrace{\mathbf{P}^{\prime \prime}\left(\left\langle s_{1}, s_{2}, 2\right\rangle,\left\langle u_{1}, u_{2}, 2\right\rangle\right)}_{=\mathbf{P}^{\prime \prime}\left(\left\langle s_{1}, s_{2}, 1\right\rangle,\left\langle u_{1}, u_{2}, 1\right\rangle\right)} \cdot \int_{0}^{t} e^{-(1+\lambda) x} \cdot \underbrace{p\left(\left\langle u_{1}, u_{2}, 2\right\rangle, t-x, n\right)}_{\geqslant p\left(\left\langle u_{1}, u_{2}, 1\right\rangle, t-x, n\right)} d x \\
& \geqslant \quad \sum_{v_{1} \in V_{1}}(1+\lambda) \cdot \mathbf{P}^{\prime \prime}\left(\left\langle s_{1}, s_{2}, 1\right\rangle,\left\langle v_{1}, s_{2}, 1\right\rangle\right) \cdot \int_{0}^{t} e^{-(1+\lambda) x} \cdot p\left(\left\langle v_{1}, s_{2}, 1\right\rangle, t-x, n\right) d x \\
& +\sum_{v_{2} \in V_{2}}(1+\lambda) \cdot \mathbf{P}^{\prime \prime}\left(\left\langle s_{1}, s_{2}, 1\right\rangle,\left\langle s_{1}, v_{2}, 1\right\rangle\right) \cdot \int_{0}^{t} e^{-(1+\lambda) x} \cdot p\left(\left\langle s_{1}, v_{2}, 1\right\rangle, t-x, n\right) d x \\
& +\sum_{\substack{u_{2} \in U_{2} \\
u_{1} \in U_{1}}}(1+\lambda) \cdot \mathbf{P}^{\prime \prime}\left(\left\langle s_{1}, s_{2}, 1\right\rangle,\left\langle u_{1}, u_{2}, 1\right\rangle\right) \cdot \int_{0}^{t} e^{-(1+\lambda) x} \cdot p\left(\left\langle u_{1}, u_{2}, 1\right\rangle, t-x, n\right) d x \\
& =p\left(\left\langle s_{1}, s_{2}, 1\right\rangle, t, n+1\right) \text {. }
\end{aligned}
$$

Lemma 4. Let $\Phi_{1}$ and $\Phi_{2}$ be CSL-formulas such that the satisfaction sets $\operatorname{Sat}\left(\Phi_{i}\right)$ are upward-closed, i.e., if $s_{1} \sqsubseteq_{m} s_{2}$ and $s_{1} \in \operatorname{Sat}\left(\Phi_{i}\right)$ then $s_{2} \in \operatorname{Sat}\left(\Phi_{i}\right)$ for $i=1,2$. Then:

$s_{1} \sqsubseteq m s_{2}$ implies $\operatorname{Prob}\left(s_{1}, \Phi_{1} \mathcal{U}^{\leqslant t} \Phi_{2}\right) \leqslant \operatorname{Prob}\left(s_{2}, \Phi_{1} \mathcal{U}^{\leqslant t} \Phi_{2}\right)$.

Proof. Using the results above and defined transformations we derive:

$$
\begin{aligned}
& \operatorname{Prob}^{\mathcal{M}}\left(s_{1}, \Phi_{1} \mathcal{U}^{\leqslant t} \Phi_{2}\right) \\
= & \{\text { Corollary } 1 \text { and Proposition } 2\}^{\prime} \operatorname{Prob}^{\mathcal{M}^{\prime \prime}}\left(\left\langle s_{1}, s_{2}, 1\right\rangle, \Phi_{1} \mathcal{U}^{\leqslant t} \Phi_{2}\right) \\
= & \{\text { Definition 10; calculus }\} \\
& \lim _{n \rightarrow \infty} p\left(\left\langle s_{1}, s_{2}, 1\right\rangle, t, n\right) \\
\leqslant & \{\text { Lemma } 6\} \\
& \lim _{n \rightarrow \infty} p\left(\left\langle s_{1}, s_{2}, 2\right\rangle, t, n\right) \\
= & \{\text { Definition } 10 ; \text { calculus }\}
\end{aligned}
$$




$$
\begin{aligned}
& \operatorname{Prob}^{\mathcal{M}^{\prime \prime}}\left(\left\langle s_{1}, s_{2}, 2\right\rangle, \Phi_{1} \mathcal{U}^{\leqslant t} \Phi_{2}\right) \\
= & \{\text { Corollary } 1 \text { and Proposition } 2\} \\
& \operatorname{Prob}^{\mathcal{M}}\left(s_{2}, \Phi_{1} \mathcal{U}^{\leqslant t} \Phi_{2}\right) .
\end{aligned}
$$

\section{References}

1. R. Alur, C. Courcoubetis and D. Dill. Model-checking in dense real-time. Inf. and Comp., 104(1): 2-34, 1993.

2. A. Aziz, V. Singhal, F. Balarin, R. Brayton and A. Sangiovanni-Vincentelli. It usually works: the temporal logic of stochastic systems. In P. Wolper (ed), ComputerAided Verification, LNCS 939, pp. 155-165, 1995.

3. A. Aziz, K. Sanwal, V. Singhal and R. Brayton. Verifying continuous time Markov chains. In R. Alur and T.A. Henzinger (eds), Computer-Aided Verification, LNCS 1102, pp. 269-276, 1996.

4. C. Baier, B.R. Haverkort, H. Hermanns and J.-P. Katoen. On the logical characterisation of performability properties. In U. Montanari et al. (eds.), Automata, Languages, and Programming, LNCS 1853, pp. 780-792, 2000.

5. C. Baier, H. Hermanns. Weak bisimulation for fully probabilistic processes. In O. Grumberg (ed), Computer-Aided Verification, LNCS 1254, pp. 119-130. 1997.

6. C. Baier, J.-P. Katoen and H. Hermanns. Approximate symbolic model checking of continuous-time Markov chains. In J.C.M. Baeten and S. Mauw (eds), Concurrency Theory, LNCS 1664, pp. 146-162, 1999.

7. C. Baier and M.I.A. Stoelinga. Norm functions for probabilistic bisimulations with delays. In J. Tyurin (ed), Found. of Software Science and Computation Structures, LNCS 1784, pp. 1-16. 2000.

8. C. Baier, B.R. Haverkort, H. Hermanns and J.-P. Katoen. Model checking continuous-time Markov chains by transient analysis. In E.A Emerson and A.P. Sistla (eds), Computer-Aided Verification, LNCS 1855, pp. 358-372, 2000.

9. C. Baier, B. Engelen, and M. Majster-Cederbaum. Deciding bisimilarity and similarity for probabilistic processes. J. of Comp. and System Sc., 60(1):187-231, 2000 .

10. M. Bernardo and R. Cleaveland. A theory of testing for Markovian processes. In C. Palamidessi (ed), Concurrency Theory, LNCS 1877, pp. 305-319, 2000.

11. M. Brown, E. Clarke, O. Grumberg. Characterizing finite Kripke structures in propositional temporal logic. Th. Comp. Sc., 59: 115-131, 1988.

12. P. Buchholz. Exact and ordinary lumpability in finite Markov chains. J. of Appl. Prob., 31: 59-75, 1994.

13. E. Clarke, M. Brown and O. Grumberg. Model checking and abstraction. ACM Tr. on Progr. Lang. and Sys., 16(5): 1512-1542, 1994.

14. E. Clarke, O. Grumberg and D. Peled. Model Checking. MIT Press, 1999.

15. P.R. D'Argenio, B. Jeannet, H.E. Jensen, and K.G. Larsen. Reachability analysis of probabilistic systems by successive refinements. In L. de Alfaro and S. Gilmore (eds), Process Algebra and Probabilistic Methods, LNCS 2165, pp. 39-56, 2001.

16. J. Desharnais, A. Edalat and P. Panangaden. A logical characterisation of bisimulation for labelled Markov processes. In IEEE Symp. on Logic in Computer Science, pp. $478-487,1998$

17. J. Desharnais and P. Panangaden. Continuous stochastic logic characterizes bisimulation of continuous-time Markov processes, 2001 (submitted for publication). (available at http://www-acaps.cs.mcgill.ca/ prakash/csl.ps). 
18. J. Desharnais, V. Gupta, R. Jagadeesan and P. Panangaden. Approximating labelled Markov processes. In IEEE Symp. on Logic in Computer Science, pp. 95$106,2000$.

19. R.J. van Glabbeek and W.P. Weijland. Branching time and abstraction in bisimulation semantics. J. ACM, 43(3): 555-600, 1996.

20. D. Gross and D.R. Miller. The randomization technique as a modeling tool and solution procedure for transient Markov chains. Oper. Res. 32(2): 343-361, 1984.

21. H. Hansson and B. Jonsson. A logic for reasoning about time and reliability. Form. Asp. of Comp. 6: 512-535, 1994.

22. H. Hermanns, J.-P. Katoen, J. Meyer-Kayser and M. Siegle. A Markov chain model checker. In S. Graf and M. Schwartzbach (eds), Tools and Algs. for the Construction and Analysis of Systems, LNCS 1785, pp. 347-362, 2000.

23. A. Jensen. Markov chains as an aid in the study of Markov processes. Skand. Aktuarietidskrift 3: 87-91, 1953.

24. B. Jonsson. Simulations between specifications of distributed systems. In J.C.M. Baeten and J.F. Groote (eds), Concurrency Theory, LNCS 527, pp. 346-360, 1991.

25. B. Jonsson and K.G. Larsen. Specification and refinement of probabilistic processes. In IEEE Symp. on Logic in Computer Science, pp. 266-277, 1991.

26. B. Jonsson, W. Yi and K.G. Larsen. Probabilistic extensions of process algebras. In J. Bergstra et al. (eds), Handbook of Process Algebra, Chapter 11, pp. 685-709, 2001.

27. J.-P. Katoen, M.Z. Kwiatkowska, G. Norman and D. Parker. Faster and symbolic CTMC model checking. In L. de Alfaro and S. Gilmore (eds), Process Algebra and Probabilistic Methods, LNCS 2165, pp. 23-38, 2001.

28. V.G. Kulkarni. Modeling and Analysis of Stochastic Systems. Chapman \& Hall, 1995.

29. K.G. Larsen and A. Skou. Bisimulation through probabilistic testing. Inf. and Comp., 94(1): 1-28, 1992.

30. R. Milner. Communication and Concurrency. Prentice-Hall, 1989.

31. A. Philippou, I. Lee, and O. Sokolsky. Weak bisimulation for probabilistic systems. In C. Palamidessi (ed), Concurrency Theory, LNCS 1877, pp. 334-349, 2000.

32. M.L. Puterman. Markov Decision Processes: Discrete Stochastic Dynamic Programming. John Wiley \& Sons, 1994.

33. R. Segala and N.A. Lynch. Probabilistic simulations for probabilistic processes. Nordic J. of Computing, 2(2): 250-273, 1995.

34. M. Silva. Private communication. 1993.

35. W.J. Stewart. Introduction to the Numerical Solution of Markov Chains. Princeton Univ. Press, 1994.

36. M.I.A. Stoelinga. Verification of Probabilistic, Real-Time and Parametric Systems. PhD Thesis, University of Nijmegen, 2002. 